\title{
Reduced dimensionality in layered quantum dimer magnets: Frustration vs. inhomogeneous condensates
}

\author{
Oliver Rösch and Matthias Vojta \\ Institut für Theoretische Physik, Universität Köln, Zülpicher Str. 77, 50937 Köln, Germany
}

(Dated: November 13, 2007)

\begin{abstract}
Motivated by recent experiments on $\mathrm{BaCuSi}_{2} \mathrm{O}_{6}$, we investigate magnetic excitations and quantum phase transitions, driven either by pressure or magnetic field, of layered dimer magnets with inter-layer frustration. We consider two scenarios, (A) a lattice with one dimer per unit cell and perfect inter-layer frustration, and (B) an enlarged unit cell with inequivalent layers, with and without perfect frustration. In all situations, the critical behavior at asymptotically low temperatures is three-dimensional, but the corresponding crossover scale may be tiny. Magnetic ordering in case (B) can be discussed in terms of two condensates; remarkably, perfect frustration renders the proximity effect ineffective. Then, the ordering transition will be generically split, with clear signatures in measurable properties. Using a generalized bond-operator method, we calculate the low-temperature magnetic properties in the paramagnetic and antiferromagnetic phases. Based on the available experimental data on $\mathrm{BaCuSi}_{2} \mathrm{O}_{6}$, we propose that scenario (B) with inequivalent layers and imperfect frustration is realized in this material, likely with an additional modulation of the inter-layer coupling along the $c$ axis.
\end{abstract}

\section{INTRODUCTION}

Magnetic quantum phase transitions (QPT) constitute an intense area of current condensed matter research. ${ }^{1-6}$ Similar to classical phase transitions, the universal critical properties of continuous QPT are determined by the symmetry of the order parameter and the number of space dimensions, $d$, but in addition the order-parameter dynamics plays a distinctive role. The criterion for meanfield behavior, being $d>4$ for classical magnetic transitions, changes to $d+z>4$ where $z$ is the dynamical exponent. Thus, realizing quantum critical behavior beyond mean-field often requires low-dimensional systems, with effectively one-dimensional (1d) or two-dimensional (2d) behavior. Such reduced dimensionality may be achieved in structured materials, consisting of chains or planes with a weak three-dimensional (3d) coupling: then, there exists an energy scale $E_{z}$ where the behavior crosses over from $3 \mathrm{~d}$ at low energies to $1 \mathrm{~d}$ or $2 \mathrm{~d}$ at higher energies.

A number of recent experiments suggested a different route to reduced dimensionality near magnetic QPT, namely through geometric frustration. One group of experiments are those on heavy-fermion metals, ${ }^{6-9}$ like $\mathrm{CeCu}_{6-x} \mathrm{Au}_{x}$ and $\mathrm{YbRh}_{2} \mathrm{Si}_{2}$, undergoing a transition towards an antiferromagnetic metallic state, with properties inconsistent with the standard theory of 3d magnetic QPT in metals. ${ }^{1-3}$ Some of the proposed theoretical explanations $^{8,10}$ are based on the assumption of the spin fluctuations being two-dimensional. Remarkably, indications for $2 \mathrm{~d}$ critical fluctuations have indeed been found in neutron scattering ${ }^{8}$ on $\mathrm{CeCu}_{6-x} \mathrm{Au}_{x}$ at the critical concentration $x_{c}=0.1$.

More recently, experiments on Mott-insulating quantum paramagnets consisting of coupled dimers of spins $1 / 2$ have been performed. Magnetic QPT can be driven either by application of a magnetic field or of pressure. ${ }^{11-18}$ The field-driven zero-temperature transition, from a paramagnet to an antiferromagnet with
XY order at a field $H=H_{c 1}$, belongs to the universality class of the dilute Bose gas, and the corresponding finite-temperature transition has been termed as "Bose-Einstein condensation of magnons". In the material $\mathrm{BaCuSi}_{2} \mathrm{O}_{6},{ }^{15,16}$ the finite-temperature transition line has been found ${ }^{18}$ to follow $T_{c} \propto\left(H-H_{c 1}\right)^{\psi}$, with a shift exponent $\psi=1$ characteristic of a two-dimensional quantum critical point (QCP), in a temperature range of $30 \mathrm{mK}<T<1 \mathrm{~K}$. As $\mathrm{BaCuSi}_{2} \mathrm{O}_{6}$ consists of layers of $\mathrm{Cu}$ dimers with a fully frustrated inter-layer coupling, i.e. a body-centered tetragonal (bct) structure of dimers, the results have been interpreted as dimensional reduction at a QCP arising from geometric frustration. ${ }^{18,19}$ We have recently shown ${ }^{20}$ that frustration, while in general not leading to $2 \mathrm{~d}$ behavior at asymptotically low energies, can strongly suppress the 3 d crossover scale $E_{z}$. More precisely, $E_{z}$ is reduced to $E_{z} \propto J_{z}^{4} / J^{3}$, compared to an unfrustrated situation with $E_{z} \propto J_{z}$; here $J$ and $J_{z}$ are characteristic in-plane and inter-plane coupling scales. (We note that, at very low energies, additional ingredients like magnetic anisotropies and coupling to nuclear spins will modify the critical behavior of a real material.)

Returning to the compound $\mathrm{BaCuSi}_{2} \mathrm{O}_{6}$, detailed neutron scattering experiments ${ }^{21}$ have revealed the existence of at least two magnetic "triplon" modes in zero field, indicating the presence of an enlarged unit cell with inequivalent dimers. In fact, the material undergoes a structural phase transition at a temperature of $100 \mathrm{~K}$, below which the magnetic layers are no longer equivalent. ${ }^{22}$ In such a situation, the application of a field will close the gap of the lowest triplon mode at $H_{c 1}$, but the condensate will be strongly inhomogeneous along the $c$ axis, and hence effectively two-dimensional. An inhomogeneous condensate for fields above $H_{c 1}$ has indeed been found in a recent NMR experiment ${ }^{23}$ on $\mathrm{BaCuSi}_{2} \mathrm{O}_{6}$ which shows the existence of (at least) two inequivalent $\mathrm{Cu}$ sites with distinct magnetizations.

This discussion suggests two distinct routes towards 

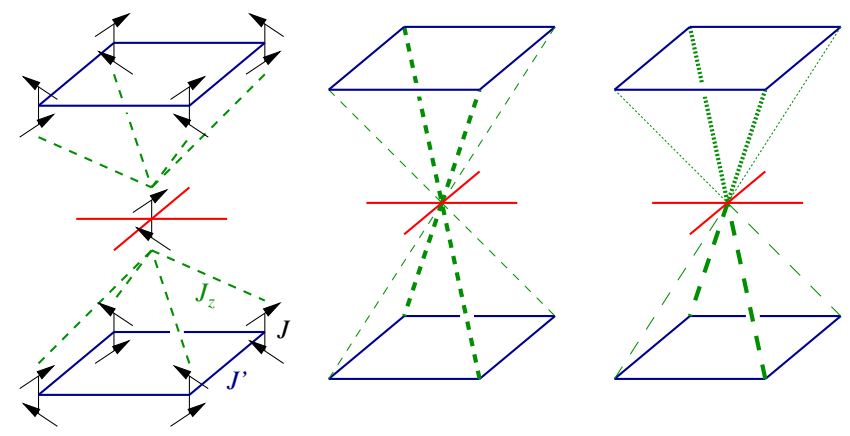

FIG. 1: a) bct lattice structure of dimers, with couplings $J$ (intra-dimer), $J^{\prime}$ (intra-layer), and $J_{z}$ (inter-layer). b,c) Illustrations of possible symmetry breaking. In b) the inter-layer couplings $J_{z}^{\Delta}$ are modulated within a unit cell such that the two diagonals are inequivalent. In c) the inter-layer couplings are further modulated along the $c$ axis, enlarging the unit cell.

critical points with reduced dimensionality in layered quantum magnets: (A) equivalent layers with perfect inter-layer frustration - this is the scenario proposed in Ref. 18 and investigated theoretically in Refs. 19,20,24; and (B) inequivalent layers ${ }^{19,21}$ - in this situation perfect frustration may still be present, but is not a required ingredient.

The purpose of this paper is twofold: First, we give a detailed discussion of situation (A), augmenting the report given in Ref. 20. Second, we study a model with two sets of inequivalent layers, i.e., two dimers per unit cell, aiming at a semi-quantitative understanding of situation (B). We shall show that qualitative differences between the cases with and without perfect frustration arise, allowing for a clear-cut experimental distinction. As in Ref. 20, our general arguments are based on an analysis of order-parameter field theories, and quantitative calculations are performed using variants of the bondoperator approach, in both the paramagnetic and antiferromagnetic phases. We shall be primarily interested in the mechanisms which suppress the $3 \mathrm{~d}$ crossover scale to small values. The physics below this scale shall not be addressed in detail, as e.g. material-dependent anisotropies (not included in our analysis) will become important.

\section{A. Main results}

In the following, we summarize our main results which strictly apply to a bet system of dimers (Fig. 1), but apply with minor modifications in general to magnetic systems with frustrated inter-layer interaction:

(i) At the magnetic quantum critical points, the asymptotic low-energy physics below a scale $E_{z}$ is in general three-dimensional. However, the crossover scale $E_{z}$ is strongly reduced compared to unfrustrated layered systems, due to either (A) frustration, or (B) a layer "mismatch" arising from inequivalent layers. (An exception is the high-field quantum phase transition between a canted

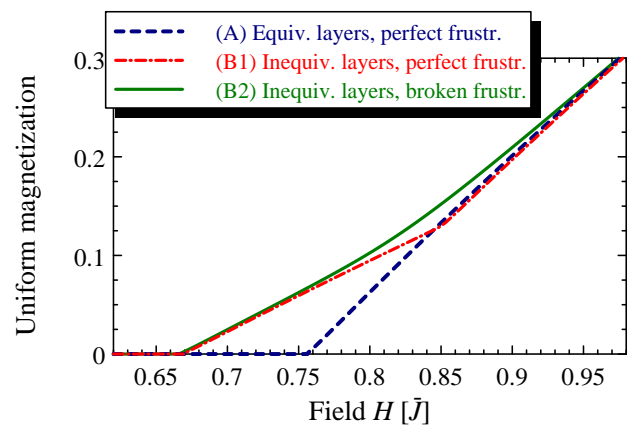

FIG. 2: Zero-temperature magnetization vs. applied field in the coupled-dimer model near $H_{c 1}$ at $T=0$, calculated using bond operators (Sec. IV), for three scenarios: (A) equivalent layers and perfect frustration (dashed), (B1) inequivalent layers and perfect frustration (dash-dot), (B2) inequivalent layers and imperfect frustration (solid). Case (B1) clearly shows the secondary transition as a kink in the magnetization curve, whereas this feature is smeared out due to the proximity effect in case (B2). Details will be discussed in Sec. VII, the parameter values are those in Figs. 9,16,17 below. We caution the reader that logarithmic corrections, not captured by our approximation, may somewhat modify the shape of the magnetization curve close to the critical field, but the existence of a sharp kink in (B1) will be unaffected.

and a fully polarized state at field $H_{c 2}$, where perfect frustration renders the low-energy physics two-dimensional, due to the lack of scattering processes at $T=0 .{ }^{19}$ )

(ii) Perfect frustration implies the absence of a linear coupling between two distinct magnetic condensates (on the even and odd layers), leading to an additional $\mathrm{Z}_{2}$ symmetry which is spontaneoulsy broken in the ordered phases. For inequivalent layers, the two magnetic condensates will be established at different critical points, i.e., the magnetic transition is generically split due to the absence of a proximity effect. This feature persists to finite temperatures and allows a distinction between perfect and imperfect frustration - in the latter case, the secondary transition is smeared out.

(iii) Applied to the field-driven QPT in $\mathrm{BaCuSi}_{2} \mathrm{O}_{6}$, our results suggest that scenario (B), with inequivalent layers and incomplete frustration, is closest to the experimental situation. To demonstrate the qualitative differences, we plot in Fig. 2 the magnetization curves close to $H_{c 1}$ for three prominent situations (equivalent layers with perfect frustration, inequivalent layers with and without perfect frustration). Finally, to reconcile the rather large magnetic proximity effect as evidenced by $\mathrm{NMR}^{23}$ with the tiny $3 \mathrm{~d}$ crossover scale ${ }^{18}$ we propose a vertically modulated inter-layer coupling which leads to a further suppression of $3 \mathrm{~d}$ behavior.

We note that a recent paper by Batista et al. ${ }^{19}$ studied in detail a hard-core boson model on a bct lattice this is the appropriate model at the $H_{c 2}$ transition, and approximately applies also in the vicinity of $H_{c 1}$, however, there it does not capture the interaction-generated $3 \mathrm{~d}$ dispersion. 


\section{B. Outline}

The bulk of the paper is organized as follows: In Sec. II we introduce the coupled-dimer model to be studied in this paper, and discuss ways of breaking the bct lattice symmetries. In Sec. III we develop an order-parameter description of the magnetically ordered phases, and present a comprehensive symmetry analysis. For quantitative calculations at zero temperature, we employ the bond-operator method, summarized in Sec. IV. Secs. V and VI contain the central results of our paper, both for scenario (A) with equivalent layers and full frustration (Sec. V) and for scenario (B) with inequivalent layers (Sec. VI). We discuss the structure of the phase diagram, the nature of the phase transitions, various energy scales relevant for thermodynamics as well as for the magnetic excitations, and zero-temperature observables like e.g. magnetizations. Finally, Sec. VII applies the results to $\mathrm{BaCuSi}_{2} \mathrm{O}_{6}$, by deriving theoretical constraints from various experimental results: This leads us to propose a scenario of inequivalent layers with partially frustrated and vertically modulated inter-layer interaction. A brief outlook concludes the paper.

Readers primarily interested in our conclusions regarding $\mathrm{BaCuSi}_{2} \mathrm{O}_{6}$ may directly jump ahead to Sec. VII (after having glanced at the notations in Sec. II).

\section{MODEL}

In this paper, we will concentrate on bct lattices, Fig. 1, consisting of two interpenetrating tetragonal subsystems. Assuming magnetic moments to be located on the sites of this lattice, with nearest-neighbor antiferromagnetic couplings, the in-plane order will be Néellike, but the coupling between adjacent layers is fully frustrated. The geometric frustration can be seen at the single-particle level: For a tight-binding model with nearest-neighbor couplings $t$ (intra-layer) and $t_{z}$ (interlayer), the single-particle dispersion is given by $\epsilon_{\vec{q}}=$ $2 t\left(\cos q_{x}+\cos q_{y}\right)+4 t_{z} \cos \left(q_{x} / 2\right) \cos \left(q_{y} / 2\right) \cos q_{z}$. For positive $t$ and small $t_{z}$, the minimum of the dispersion is at in-plane wavevector $\vec{q}_{\|}=(\pi, \pi)$, where it is independent of $q_{z}$ - this is the result of inter-layer frustration.

With the application to coupled-dimer materials like $\mathrm{BaCuSi}_{2} \mathrm{O}_{6}$ in mind, we will consider a Heisenberg Hamiltonian for dimers of spins 1/2. We decompose the Hamiltonian into in-plane and inter-plane parts,

$$
\mathcal{H}=\mathcal{H}_{\|}+\mathcal{H}_{z}
$$

The in-plane part is unfrustrated and reads

$$
\begin{aligned}
\mathcal{H}_{\|} & =\sum_{i n} J_{n} \vec{S}_{i n 1} \cdot \vec{S}_{i n 2}+\sum_{\langle i j\rangle n m} J_{n}^{\prime} \vec{S}_{i n m} \cdot \vec{S}_{j n m} \\
& -\vec{H} \cdot \sum_{i n m} \vec{S}_{i n m}
\end{aligned}
$$

where $m=1,2$ labels the spins of each dimer, $i, j$ are the dimer site indices in each layer, and $n$ the layer index. $J_{n}$ and $J_{n}^{\prime}$ are the antiferromagnetic intra-dimer and inplane inter-dimer couplings, and $\vec{H}$ is an external uniform field.

A rather general form of the inter-layer coupling reads

$$
\begin{aligned}
\mathcal{H}_{z} & =\sum_{i \Delta n m m^{\prime}} J_{z}^{n m m^{\prime} \Delta} \vec{S}_{i n m} \cdot \vec{S}_{i+\Delta, n+1, m^{\prime}} \\
& +\sum_{i n m m^{\prime}} J_{z z}^{n m m^{\prime}} \vec{S}_{i n m} \cdot \vec{S}_{i, n+2, m^{\prime}}
\end{aligned}
$$

$J_{z}$ is the frustrated coupling between adjacent layers, whereas $J_{z z}$ represents an unfrustrated coupling between second-neighbor layers. The $\sum_{\Delta}$ runs over four spatial diagonals $(\Delta=1, \ldots, 4)$ such that the sites $(i n)$ and $(i+\Delta, n+1)$ are nearest neighbors in $z$ direction, i.e., with distance $( \pm a / 2, \pm a / 2, c)$ where $a$ and $c$ are the lattice constants (which are set to unity in the following). Note that $J_{z}^{n m m^{\prime} \Delta}$ has some specific inter-dimer structure given by the $\mathrm{mm}^{\prime}$ dependence, and frustration can be broken due to the $\Delta$ dependence, see below. The $\mathrm{mm}^{\prime}$ dependence requires discussion, as different physical processes are determined by different combinations of $J_{z}^{m m^{\prime}}$ : The "bare dispersion" of the triplet excitations is determined by $J_{2 z}=J_{z}^{11}+J_{z}^{22}-J_{z}^{12}-J_{z}^{21}$, whereas the combination $J_{4 z}=J_{z}^{11}+J_{z}^{22}+J_{z}^{12}+J_{z}^{21}$ enters in the interaction vertex. If we label the lower (upper) spin in each dimer with 1 (2), Fig. 1a, the geometry suggests that $J_{z}^{21}$ is dominant and antiferromagnetic. Hence, in what follows we shall assume $-J_{2 z}=J_{4 z} \equiv J_{z}>0$ unless otherwise noted.

Occasionally we will refer to a possible biquadratic inter-layer exchange term:

$$
\mathcal{H}_{\text {coll }}=\sum_{i \Delta n m m^{\prime}} J_{\text {coll }}^{n m m^{\prime} \Delta}\left(\vec{S}_{i n m} \cdot \vec{S}_{i+\Delta, n+1, m^{\prime}}\right)^{2} .
$$

As we have shown in Ref. 20, an effective secondneighbor inter-layer coupling is always generated $\left(\propto J_{z}^{4}\right)$ through interaction processes, even if a "bare" $J_{z z}$ is absent in an idealized model Hamiltonian. (The same applies to the biquadratic term $J_{\text {coll }}$, with $J_{\text {coll }} \propto-J_{z}^{2}$.) In the microscopic calculations to be presented in this paper, we set $J_{z z}=J_{\text {coll }}=0$ unless otherwise noted.

\section{A. Equivalent layers}

For an ideal bct lattice structure, all layers are equivalent, $J_{n}=J, J_{n}^{\prime}=J^{\prime}$, and the inter-layer coupling is fully frustrated, i.e., the four diagonal bonds are equal, $J_{z}^{n m m^{\prime} \Delta}=J_{z}^{m m^{\prime}}$. Importantly, the second-neighbor coupling in $z$ direction is allowed by symmetry, ${ }^{20} J_{z z} \neq 0$ in general.

Distortions of the ideal bct lattice may or may not enlarge the unit cell. In the latter case, particularly interesting are distortions which break the perfect interlayer frustration. Those correspond to a $\Delta$ dependence of 
$J_{z}^{n m m^{\prime} \Delta}$ which renders the four links between one site and its neighbors in $z$ direction inequivalent. The simplest symmetry breaking leads to different couplings along the two diagonals, see Fig. 1b.

\section{B. Inequivalent layers}

Various lattice distortions can occur which enlarge the unit cell. This is likely the case in the low-temperature phase of $\mathrm{BaCuSi}_{2} \mathrm{O}_{6}$, which, however, to our knowledge, has not been fully characterized to date. For simplicity and motivated by the $\mathrm{BaCuSi}_{2} \mathrm{O}_{6}$ neutron scattering results, ${ }^{21}$ we will assume that the distortions preserve the tendency towards commensurate in-plane ordering at wavevector $(\pi, \pi)$, but we allow for layer-dependent in-plane couplings $J, J^{\prime}$ and possibly broken inter-layer frustration.

Importantly, within the two sets of "even" and "odd" layers (i.e. layers with even and odd $n$ ) a unfrustrated (albeit small) coupling will be present through $J_{z z}$, irrespective of the presence or absence of inter-layer frustration. Thus the simplest and physically most interesting scenario is one where all even planes are identical, as are the odd ones, i.e., we have a lattice still consisting of two tetragonal subsystems, labeled $A$ ( $n$ even) and $B(n$ odd). Then the couplings take the form:

$$
J_{2 n}=J_{A}, J_{2 n}^{\prime}=J_{A}^{\prime}, J_{2 n+1}=J_{B}, J_{2 n+1}^{\prime}=J_{B}^{\prime}
$$

This also implies two different second-neighbor vertical couplings, $J_{z z}^{2 n}=J_{z z}^{A}, J_{z z}^{2 n+1}=J_{z z}^{B}$.

The coupling between adjacent layers may still have the full symmetry, $J_{z}^{n m m^{\prime} \Delta}=J_{z}^{m m^{\prime}}$, implying perfect frustration. Alternatively, the distortion can break the frustration. The simplest situation with inequivalent diagonals, Fig. 1b, can be described by $J_{z}$ couplings according to

$$
\begin{aligned}
J_{z}^{2 n m m^{\prime} \Delta} & =\left\{\begin{array}{ll}
J_{z A 1}^{m m^{\prime}} & \Delta \text { odd } \\
J_{z A 2}^{m m^{\prime}} & \Delta \text { even }
\end{array},\right. \\
J_{z}^{2 n+1, m m^{\prime} \Delta} & =\left\{\begin{array}{ll}
J_{z B 1}^{m m^{\prime}} & \Delta \text { odd } \\
J_{z B 2}^{m m^{\prime}} & \Delta \text { even }
\end{array} .\right.
\end{aligned}
$$

Here we have also allowed for a modulation of the $J_{z}$ coupling along the $c$ axis, as illustrated in Fig. 1c - this will turn out to be relevant for our analysis of $\mathrm{BaCuSi}_{2} \mathrm{O}_{6}$. (Note that $J_{A} \neq J_{B}, J_{A}^{\prime} \neq J_{B}^{\prime}$ on the one hand and $J_{z A} \neq J_{z B}$ on the other hand break different mirror symmetries of the bct lattice.)

All scenarios included above lead to a unit cell containing no more than two dimers (one from the even and one from the odd layers). A further enlarged unit cell, i.e., multiple dimers within each family of layers, only lead to quantitative modifications of the overall physics, and will be discussed towards the end of the paper.

\section{Phases}

To set the stage, we sketch the phase diagram of the coupled-dimer model $\mathcal{H}$ (ignoring the complications arising from structural distortions of the ideal bct lattice). At zero temperature the phase diagram is similar to that of the well-studied bilayer Heisenberg model, ${ }^{25-29}$ see e.g. Fig. 1 of Ref. 27.

For $J \gg J^{\prime},\left|J_{z}\right|$, the zero-field ground state of $\mathcal{H}$ is a paramagnetic singlet, with elementary gapped triplet excitations. The number of excitation branches is equal to the number of dimers per unit cell.

If $J^{\prime}$ dominates, an antiferromagnetic phase with broken $\mathrm{SU}(2)$ symmetry and in-plane ordering wavevector $(\pi, \pi)$ is established. In the case of perfect interlayer frustration, layers with classical moments would be uncoupled, leaving an infinite ground-state manifold. This degeneracy is lifted for quantum spins by a zerotemperature order-from-disorder mechanism, which establishes true $3 \mathrm{~d}$ order (Sec. V B). In contrast, for large $\left|J_{z}\right|$ the in-plane order is ferromagnetic, with the interplane order depending on the sign of $J_{z}$.

Applying a field to the large- $J$ quantum paramagnet leads to a Zeeman splitting of the triplet excitations. At a critical field $H_{c 1}$, the gap of the lowest mode closes, and a quantum phase transition to a gapless canted phase occurs. (For modifications due to inequivalent dimers see Sec. VI.) The canted phase has a broken U(1) symmetry, corresponding to XY order perpendicular to the field. Upon further increasing the field, the system is driven into a fully polarized state at $H_{c 2}$.

\section{SYMMETRIES AND ORDER-PARAMETER FIELD THEORY}

In this section, we present the ingredients for a LandauGinzburg-Wilson description of the degrees of freedom of the coupled-dimer model $\mathcal{H}$. We start with antiferromagnetic fluctuations on a paramagnetic background.

A remark of caution is in order: The formal derivation of an effective theory for the staggered magnetization involves integrating out the degrees of freedom associated with the uniform magnetization, see e.g. Ref. 4. (The "local" staggered and uniform magnetizations correspond to $\vec{S}_{1}-\vec{S}_{2}$ and $\vec{S}_{1}+\vec{S}_{2}$, respectively, in terms of the two spins $\vec{S}_{1}, \vec{S}_{2}$ of a dimer in $\mathcal{H}(1)$.) However, there exist processes which are strictly forbidden within the effective theory for the staggered magnetization, but exist in the full theory. One example is the two-particle (as opposed to three-particle) decay of triplons discussed recently in Refs. 30,31; another example is the effective vertical second-neighbor hopping of order $J_{z}^{4}$ in the model $\mathcal{H}(1)$, to be described below in Sec. V A. 


\section{A. Antiferromagnetic order parameter}

A continuum description of antiferromagnetic fluctuations on bct lattices requires care due to the geometric frustration. In-plane magnetic fluctuations are strong and centered around wavevector $\vec{Q}=(\pi, \pi)$, but the effective inter-layer coupling is frustrated and weak. Hence there will be a large regime of energies or temperatures with no well-defined order along the $c$ axis. Therefore we only take the continuum limit w.r.t. the in-plane coordinates, but keep the discrete layer index $n$. We thus define an order parameter $\vec{\phi}_{n}\left(\vec{r}_{\|}\right)$, where $\vec{r}_{\|}$is the in-plane coordinate, with the local magnetization operator given by $\vec{m}_{n}\left(\vec{r}_{\|}\right)=\exp \left(i \vec{Q} \cdot \vec{r}_{\|}\right) \vec{\phi}_{n}\left(\vec{r}_{\|}\right)$. As outlined above, we will allow for unequal layers, but assume a two-dimer unit cell. Hence, we will use the labels $A(B)$ for even (odd) layers and the respective parameters.

The full $\phi^{4}$ theory for the magnetic fluctuations is

$$
\mathcal{S}_{\phi}=\mathcal{S}_{2 A}+\mathcal{S}_{2 B}+\mathcal{S}_{4 A}+\mathcal{S}_{4 B}+\mathcal{S}_{\mathrm{dyn}}+\mathcal{S}_{A B}
$$

The first two terms, $\mathcal{S}_{2 A}+\mathcal{S}_{2 B}$, contain the Gaussian description of the unfrustrated magnetism of the two tetragonal subsystems:

$$
\begin{array}{r}
\mathcal{S}_{2 A}=\int d \tau d^{2} k_{\|} \sum_{n \text { even }}\left[\left(m_{\phi A}+c_{A}^{2} k_{\|}^{2}\right) \vec{\phi}_{n A}^{2}\left(\vec{k}_{\|}\right)\right. \\
\left.+\eta_{A}^{\prime} \vec{\phi}_{n A} \cdot \vec{\phi}_{n+2, A}\right]
\end{array}
$$

the action $\mathcal{S}_{2 B}$ for odd layers is obtained by $A \rightarrow B$. Here $\vec{\phi}\left(\vec{k}_{\|}\right)$is the order-parameter field after in-plane Fourier transformation, the momentum $\vec{k}_{\|}$is now measured relative to the ordering wavevector $(\pi, \pi)$, and $c$ is a velocity. Further, $\eta^{\prime}$ represents the unfrustrated vertical second-neighbor coupling. From the microscopic model one reads off the bare value $\eta^{\prime} \sim J_{z z}$; however, as shown in Ref. 20, interaction effects cause a non-zero $\eta^{\prime}$ even for vanishing bare $J_{z z}$, see Sec. VA. The mass $m_{\phi}$ is the control parameter of the zero-field transition; a negative (renormalized) mass $m_{\phi A}$ leads to a magnetic condensate with $\left\langle\vec{\phi}_{A}\right\rangle \neq 0$. (The coupling between $\vec{\phi}_{A}$ and $\vec{\phi}_{B}$ will be discussed below.) The terms $\mathcal{S}_{4 A}, \mathcal{S}_{4 B}$ contain the local quartic self-interaction,

$$
\mathcal{S}_{4 A}=u_{0} \int d \tau d^{2} r_{\|} \sum_{n \text { even }}\left[\vec{\phi}_{n A}^{2}\left(\vec{r}_{\|}\right)\right]^{2} .
$$

Finally, $\mathcal{S}_{\text {dyn }}$ encodes the dynamics of the spin fluctuations: ${ }^{4}$

$$
\mathcal{S}_{\mathrm{dyn}}=\int d \tau d^{2} r_{\|} \sum_{n}\left(\partial_{\tau} \vec{\phi}_{n}-i \vec{H} \times \vec{\phi}_{n}\right)^{2}
$$

In zero field, there is only a second-order time derivative, the dynamical exponent is $z=1$, and the modes are triply degenerate. In contrast, in finite field we have $z=$ 2 , and the modes are Zeeman-split according to $\omega \rightarrow \omega-$ $\alpha H$, where $\alpha=+, 0,-$ and the,+- modes correspond to $\phi_{x} \pm i \phi_{y}$ (assuming the field to be in $z$ direction).

Let us now turn to the frustrated coupling between adjacent layers, described by $\mathcal{S}_{A B}$. To this end, we repeat the central symmetry argument for the perfectly frustrated geometry, already given in Ref. 20. Apart from spin rotation and space inversion symmetry, the system is also invariant under 90-degree in-plane rotations, however, the geometry dictates that this is accompanied by a relative sign change of the order parameter in two neighboring planes (!):

$$
k_{x} \rightarrow k_{y}, k_{y} \rightarrow-k_{x}, \vec{\phi}_{n} \rightarrow(-1)^{n} \vec{\phi}_{n} .
$$

This symmetry strongly constrains the allowed interlayer coupling terms in the case of perfect frustration. A general form of the inter-layer coupling, including quadratic and quartic terms, is

$$
\begin{aligned}
\mathcal{S}_{A B} & =\int d \tau d^{2} k_{\|} \sum_{n}\left[\kappa \vec{\phi}_{n} \cdot \vec{\phi}_{n+1}+\eta k_{x} k_{y} \vec{\phi}_{n} \cdot \vec{\phi}_{n+1}\right. \\
& \left.+u_{1}\left(\vec{\phi}_{n} \cdot \vec{\phi}_{n+1}\right)^{2}+u_{2} \vec{\phi}_{n}^{2} \vec{\phi}_{n+1}^{2}\right]
\end{aligned}
$$

(To avoid clutter of notation, we have assumed the couplings to be vertically unmodulated; a corresponding generalization is straightforward.) Clearly, the $\kappa$ term - actually corresponding to unfrustrated inter-layer coupling - is incompatible with the symmetry (11), whereas the other terms are compatible. Thus, perfect frustration implies $\kappa=0$. The $\eta$ term represents single-particle hopping in the presence of frustration, i.e., it vanishes for $k_{x}=0$ or $k_{y}=0$; the form of this hopping term can be directly obtained from expanding the tight-binding dispersion on the bct lattice near in-plane wavevector $(\pi, \pi)$. From the microscopic model (1) one reads off $\eta \propto J_{z}$.

Further, $u_{1}$ and $u_{2}$ represent density interactions between adjacent layers. An important role - in particular in the ordered phase - is played by the $u_{1}$ term: in the presence of perfect frustration this is the leading coupling between adjacent layers at $k_{\|}=0$ which is sensitive to spin directions. Negative $u_{1}$ stabilizes collinear spin correlations between adjacent planes, while positive $u_{1}$ favors orthogonal $\vec{\phi}_{A}$ and $\vec{\phi}_{B}$. Comparing with the microscopic model (1), it is obvious that the $u_{1}$ term only arises at order $J_{z}^{2}$, in fact $u_{1} \propto-J_{z}^{2} / J$, see Sec. V A. (These statements hold in the absence of a bare $\mathcal{H}_{\text {coll }}$, otherwise $u_{1} \propto J_{\text {coll }}$ dominates.)

\section{B. Full frustration: $\mathbf{Z}_{2}$ symmetry and Ising bond order parameter}

As the bilinear magnetic coupling between adjacent layers, $\vec{\phi}_{n} \cdot \vec{\phi}_{n+1}$, is suppressed by a prefactor of $k_{x} k_{y}$ in the presence of perfect frustration, there is no linear coupling between the condensates $\left\langle\vec{\phi}_{A}\right\rangle$ and $\left\langle\vec{\phi}_{B}\right\rangle$ on the even and odd layers in an antiferromagnetically ordered phase. Instead, the dominant $A-B$ coupling is given by 
the biquadratic term $\propto u_{1}$, which will select collinear or orthogonal correlations between the two condensates, but will always leave a $Z_{2}$ degeneracy intact, corresponding to a spin inversion in every second plane. This $Z_{2}$ symmetry corresponds to a true symmetry for the antiferromagnet on the bct lattice, and will be spontaneously broken in the ordered phase.

For an undistorted lattice, it is then useful to introduce a local Ising order parameter $\Psi_{n+1 / 2}$ which is conjugate to $\vec{\phi}_{n} \cdot \vec{\phi}_{n+1}$ and lives at zero in-plane wavevector. $\Psi$ transforms as a singlet under $\mathrm{SU}(2)$ spin rotations, and hence can be described by an unfrustrated $\Psi^{4}$ theory. Assuming a single-dimer unit cell, a plausible form is:

$$
\begin{aligned}
\mathcal{S}_{\psi}= & \int d \tau d^{2} k_{\|}\left[\sum_{n}\left(m_{\Psi}+c_{\Psi}^{2} k_{\|}^{2}\right) \Psi_{n+1 / 2}^{2}\left(\vec{k}_{\|}\right)\right. \\
& \left.+\eta_{\Psi} \Psi_{n-1 / 2} \Psi_{n+1 / 2}\right]+\mathcal{S}_{\Psi 4}+\mathcal{S}_{\Psi \mathrm{dyn}}
\end{aligned}
$$

where $\mathcal{S}_{\Psi 4}$ is again a quartic self-interaction, and the dynamic term $\mathcal{S}_{\Psi \text { dyn }}$ contains a second-order time derivative. The physical content of $\Psi$ is encoded in its interaction with $\vec{\phi}$, where the leading term is trilinear:

$$
\mathcal{S}_{\phi \Psi}=\lambda \int d \tau d^{2} k_{\|} \sum_{n} \Psi_{n+1 / 2} \vec{\phi}_{n} \cdot \vec{\phi}_{n+1}
$$

with $\lambda$ a coupling constant. (Additional couplings $\Psi \vec{\phi}_{n}^{2}$ etc. do not modify the physics to be discussed below.)

Eq. (14) shows that the condensation of $\Psi$ induces an unfrustrated vertical hopping through the term $\lambda\langle\Psi\rangle \vec{\phi}_{n}$. $\vec{\phi}_{n+1}$, i.e., it breaks the frustration. Microscopically, $\Psi$ condensation is equivalent to spontaneous bond order, modulating the vertical magnetic couplings $J_{z}^{\Delta}$ within each unit cell, as illustrated in Fig. 1b. Within a purely magnetic model, $\Psi$ can be understood as a singlet bound state of two $\vec{\phi}$ quanta, implying that $m_{\Psi}$ is essentially given by $2 m_{\phi}$, plus a correction arising due to an attraction or repulsion of $\vec{\phi}$ quanta from $\mathcal{S}_{A B}(12)$.

In the presence of phononic degrees of freedom, bond order causes lattice distortions, hence $\Psi$ condensation is a structural phase transition. $\Psi$ may condense either uniformly or with a non-trivial modulation along the $c$ axis. (In principle, the action $\mathcal{S}_{\Psi}$ could be dominated by phonon effects - this will not be considered.)

Let us now discuss the implications for the phase diagram of the spin model on the ideal bct lattice: The full theory $\mathcal{S}_{\phi}+\mathcal{S}_{\Psi}+\mathcal{S}_{\phi \Psi}$ admits two distinct scenarios: (i) A single transition driven by the condensation of $\phi$ - here, the coupling $\lambda$ generates a non-zero expectation value for $\Psi$ as well, because the $u_{1}$ term in $\mathcal{S}_{A B}$ leads to non-zero $\left\langle\vec{\phi}_{n} \cdot \vec{\phi}_{n+1}\right\rangle$. (ii) Two transitions: First, $\Psi$ condenses, which modifies the quadratic part of the $\phi$ action, relieving the frustration, and $\phi$ orders in a second, subsequent transition. For the microscopically relevant parameters, we find that situation (i) is generically realized, see Sec. V C 2.

\section{Canted magnetism near $H_{c 1}$}

Near the critical field $H_{c 1}$, only the lowest of the Zeeman-split triplet modes is relevant for the low-energy behavior. This lowest mode is $\Phi=\phi_{x}+i \phi_{y}$, and the critical theory can be formulated using a single complex scalar field $\Phi$ with canonical boson dynamics and a mass (i.e. chemical potential) $\sim\left(H_{c 1}-H\right)$. Condensation of $\Phi$ breaks a U(1) symmetry and leads to spontaneous order perpendicular to the field direction: the transverse staggered magnetization is given by $\langle\Phi\rangle$, whereas the longitudinal uniform magnetization is $\left\langle\Phi^{*} \Phi\right\rangle$. Thus, the physics near $H_{c 1}$ is that of a dilute Bose gas. ${ }^{4}$ (Complications again arise from the additional $\mathrm{Z}_{2}$ symmetry, the detailed discussion will be given in Sec. VD.)

The above symmetry analysis in terms of $\vec{\phi}$ continues to apply, with the change that $\vec{\phi}$ is now a two-component vector representing the transverse staggered magnetization. However, care is needed when associating effective with microscopic couplings, as e.g. $\vec{\phi}^{2}$ now carries a uniform magnetization and hence couples linearly to a field. (Technically, this arises because the degrees of freedom of the uniform magnetization are no longer gapped.)

\section{Canted magnetism near $H_{c 2}$}

At high fields the ground state of the system is fully polarized, and the elementary excitations are bosonic spin-flip quasiparticles. Upon decreasing the field, those will condense at $H_{c 2}$, leading to a canted phase which is continuously connected to the canted phase established above $H_{c 1}$. Hence, the order-parameter description is identical to the one near $H_{c 1}$ : The order parameter is a canonical boson $\Phi$, now with a mass $\sim\left(H-H_{c 2}\right)$. The transverse staggered magnetization is again given by $\langle\Phi\rangle$, whereas the uniform magnetization is $M_{\mathrm{sat}}-\left\langle\Phi^{*} \Phi\right\rangle$.

\section{E. Phase transitions}

Most of the quantum phase transitions discussed below are at or above their upper-critical dimension (which the exception of the Ising and $\mathrm{O}(3)$ transitions in $d=2$ ), thus the critical exponents are known. The shift exponent $\psi$ of the finite-temperature phase boundary is given by the product of correlation length and dynamical exponents, $\nu z$, if the QPT is below its upper critical dimension; otherwise it can be obtained from the temperature dependence of the Hartree diagram determining the mass shift of the order parameter, ${ }^{4}$ and is given by $\psi=z /(d+z-2)$ ( $z=2$ for a BEC transition).

In this paper, we intend to estimate relevant energy scales and to obtain the overall behavior of observables, primarily at zero temperature away from the phase transitions. To this end, we employ bare as well as selfconsistent perturbation theory. These methods may 
break down near criticality, and we comment on this below.

The finite- $T$ regime close to the ordering temperature is more difficult: The interplay of the frustration-related order-from-disorder mechanism and the Mermin-Wagner theorem is delicate, see Sec. VE. A reliable treatment of the finite-temperature transitions, including an estimate of $T_{\mathrm{N}}$, is beyond the scope of the paper.

\section{BOND-OPERATOR THEORY}

For a quantitative study of the coupled-dimer Heisenberg model (1) we apply the bond-operator approach of Sachdev and Bhatt, ${ }^{32}$ with extensions proposed by Kotov et $a .^{28}$ and by Sommer et al. ${ }^{29}$ While this method can in principle be applied at finite temperatures, ${ }^{33}$ we will restrict the explicit calculations to $T=0$.

In this section, we present the formalism for a situation with equivalent dimers, i.e. a bct lattice with a single-site unit cell (with lattice sites denoted by $i$ ). The generalization to inequivalent dimers is straightforward, and we shall refrain from showing the lengthy equations.

\section{A. Paramagnetic phase: Harmonic approximation}

The four states of a dimer $i$ can be represented using bosonic "bond" operators $\left\{s_{i}^{\dagger}, t_{i \alpha}^{\dagger}\right\} \quad(\alpha=x, y, z)$, which create the dimer states out of a fictitious vacuum. Explicitly (and omitting the site index $i$ ), $|s\rangle=s^{\dagger}|0\rangle$, $|\alpha\rangle=t_{\alpha}^{\dagger}|0\rangle$, where $|s\rangle=(|\uparrow \downarrow\rangle-|\downarrow \uparrow\rangle) / \sqrt{2},|x\rangle=$ $(-|\uparrow \uparrow\rangle+|\downarrow \downarrow\rangle) / \sqrt{2},|y\rangle=i(|\uparrow \uparrow\rangle+|\downarrow \downarrow\rangle) / \sqrt{2},|z\rangle=(|\uparrow \downarrow\rangle+$ $|\downarrow \uparrow\rangle) / \sqrt{2}$. The Hilbert space dimension is conserved by imposing the constraint $s_{i}^{\dagger} s_{i}+\sum_{\alpha} t_{i \alpha}^{\dagger} t_{i \alpha}=1$ on every site $i$. (In the presence of a Zeeman field, a rotated triplet basis with $t_{i+}^{\dagger}=\left(t_{i x}^{\dagger}+i t_{i y}^{\dagger}\right) / \sqrt{2}, t_{i-}^{\dagger}=\left(t_{i x}^{\dagger}-i t_{i y}^{\dagger}\right) / \sqrt{2}$, $t_{i 0}^{\dagger}=t_{i z}^{\dagger}$ is useful as well.)

The Heisenberg Hamiltonian (1) can now be formulated in terms of the bond operators $\left\{s_{i}, t_{i \alpha}\right\}$, for details see Refs. 28,32,34. To treat the paramagnetic phase, the following re-interpretation of the formalism is useful: ${ }^{28}$ Starting from a background product state of singlets on all dimers, $\left|\psi_{0}\right\rangle=\prod_{i} s_{i}^{\dagger}|0\rangle$, the operators $t_{i \alpha}^{\dagger}$ can be viewed as creating local triplet excitations in the singlet background. (Formally, this is achieved by setting $s_{i}=s_{i}^{\dagger}=1$ which implies $t_{i \alpha}^{\dagger} s_{i} \rightarrow t_{i \alpha}^{\dagger}$.) The constraint then takes the form $\sum_{\alpha} t_{i \alpha}^{\dagger} t_{i \alpha} \leq 1$. (So far, the procedure is exact.)

Upon expressing $\mathcal{H}(1)$ in bond operators, products of two spin operators convert into terms with two, three, and four triplet operators, $\mathcal{H}=\mathcal{H}_{2}+\mathcal{H}_{3}+\mathcal{H}_{4}$; the biquadratic spin term in $\mathcal{H}_{\text {coll }}$ (4) contains up to 8 triplets. For the ideal bct structure and an external field in $z$ di- rection, the bilinear part $\mathcal{H}_{2}$ reads:

$$
\mathcal{H}_{2}=\sum_{\vec{q} \alpha}\left\{\left(A_{\vec{q}}-\alpha H\right) t_{\vec{q} \alpha}^{\dagger} t_{\vec{q} \alpha}+\frac{B_{\vec{q}}}{2}\left(t_{\vec{q} \alpha} t_{-\vec{q} \bar{\alpha}}+\text { h.c. }\right)\right\}
$$

with $\alpha=+, 0,-, \bar{\alpha}=-\alpha, A_{\vec{q}}=J+B_{\vec{q}}, B_{\vec{q}}=2 J^{\prime} \gamma_{\vec{q} \|}+$ $2 J_{2 z} \gamma_{\vec{q} z}$ and

$$
\begin{aligned}
\gamma_{\vec{q} \|} & =\left(\cos q_{x}+\cos q_{y}\right) / 2, \\
\gamma_{\vec{q} z} & =\cos \left(q_{x} / 2\right) \cos \left(q_{y} / 2\right) \cos q_{z} .
\end{aligned}
$$

The coupling constant $J_{2 z}$ appearing in $A_{\vec{q}}, B_{\vec{q}}$ is $J_{2 z}=$ $J_{z}^{11}+J_{z}^{22}-J_{z}^{12}-J_{z}^{21}$. The $3 \mathrm{~d}$ momentum $\vec{q}$ runs over the Brillouin zone of the bct lattice, spanned by the primitive translations $\hat{\vec{q}}_{1}=(2 \pi, 0,-\pi), \hat{\vec{q}}_{2}=(0,2 \pi,-\pi)$, $\hat{\vec{q}}_{3}=(0,0,2 \pi)$ in reciprocal space.

The harmonic (or linearized) approximation consists in treating only the bilinear part $\mathcal{H}_{2}$ of the Hamiltonian; both the hard-core constraint and the higher-order terms $\mathcal{H}_{3}, \mathcal{H}_{4}$ (given in App. A) are neglected. With the Bogoliubov transformation $t_{\vec{q} \alpha}=u_{\vec{q}} \tau_{\vec{q} \alpha}+v_{\vec{q}} \tau_{-\vec{q}, \bar{\alpha}}^{\dagger}$ the Hamiltonian $\mathcal{H}_{2}$ can be diagonalized, with eigenvalues

$$
\omega_{\vec{q} \alpha}=\sqrt{A_{\vec{q}}^{2}-B_{\vec{q}}^{2}}-\alpha H
$$

and Bogoliubov coefficients

$$
u_{\vec{q}}^{2}, v_{\vec{q}}^{2}= \pm \frac{1}{2}+\frac{A_{\vec{q}}}{2 \omega_{\vec{q} 0}}, \quad u_{\vec{q}} v_{\vec{q}}=-\frac{B_{\vec{q}}}{2 \omega_{\vec{q} 0}} .
$$

In this linearized bond-operator theory, interactions between the order-parameter fluctuations - represented by triplet quasiparticles - are ignored.

For $\left|J_{2 z}\right| / J<2$ the dispersion minimum of the magnetic modes is at $\vec{q}_{\|}=(\pi, \pi)$. There, $\omega_{\vec{q} \alpha}$ is independent of $q_{z}$ due to frustration - hence effectively twodimensional. Expanding near $(\pi, \pi)$ yields:

$$
\omega_{\vec{q} 0}=\Delta+\frac{c^{2}}{\Delta}\left(k_{\|}^{2}+\eta k_{x} k_{y} \cos q_{z}\right)
$$

where $\vec{k}_{\|}=\vec{q}_{\|}-(\pi, \pi)$ and $\eta=-J_{2 z} /\left(2 J^{\prime}\right)$. The spin gap and velocity are given by $\Delta=\sqrt{J\left(J-4 J^{\prime}\right)}$ and $c=\sqrt{J J^{\prime} / 2}$.

For larger unit cells, multiple triplon operators are introduced, and the Bogoliubov transformation needs to be performed numerically.

\section{B. Beyond the harmonic approximation}

Triplon interactions effects are important to lift the degeneracy of the dispersion along $\left(\pi, \pi, q_{z}\right)$. The most important interaction correction arises from the hard-core constraint, which is conveniently implemented using an infinite on-site repulsion between the bosons: ${ }^{28}$

$$
\mathcal{H}_{U}=U \sum_{i \alpha \beta} t_{i \alpha}^{\dagger} t_{i \beta}^{\dagger} t_{i \alpha} t_{i \beta}, \quad U \rightarrow \infty .
$$


As proposed by Kotov et al. ${ }^{28}$, this hard-core term can be treated by a ladder summation of scattering diagrams. together with a self-consistent one-loop approximation for the self energy, Fig. 1 of Ref. 28. This method is also known as Brueckner approach in particle theory, the small parameter being the density of triplet bosons, which at zero temperature is given by $\sum_{\vec{q} \alpha} v_{\vec{q} \alpha}^{2}$.

Here, we have employed the formalism of Ref. 28 at finite fields (App. A). In the present problem, it is not sufficient to treat the quartic terms in $\mathcal{H}_{4}$ in a mean-field (Hartree-Fock) approximation: processes of second order in $\mathcal{H}_{4}$ are needed to obtain the leading contribution $\propto J_{z}^{4}$ to the unfrustrated second-neighbor hopping in $z$ direction. The calculations involve the self-consistent solution of integral equations and are performed numerically on lattices with up to $16^{3}$ sites.

The approach of Ref. 28 has been shown to give results in good quantitative agreement with Quantum Monte Carlo and series-expansion methods, regarding e.g. phase boundaries and magnetic excitations of the bilayer Heisenberg model. Although the Brueckner method contains a re-summation of an infinite series of diagrams, it is not designed to capture critical behavior beyond mean-field.

\section{Ordered phases}

The bond-operator method can be generalized to magnetically ordered states by taking into account the appropriate condensate. Technically, an expansion is then performed around a symmetry-broken product state $\left|\psi_{0}\right\rangle$ which replaces the singlet state. As demonstrated by Sommer et al. ${ }^{29}$ a consistent description of the fluctuations is obtained by applying a harmonic approximation after a rotation of the basis vectors in the fourdimensional Hilbert space of each dimer.

Here we adopt the formalism of Ref. 29, briefly summarized in the following, to the bct lattice geometry. The rotated basis operators, replacing $\left\{s_{i}^{\dagger}, t_{i \alpha}^{\dagger}\right\}$, are

$$
\begin{aligned}
\tilde{s}_{i}^{\dagger} & =\frac{1}{\sqrt{1+\lambda^{2}}}\left[s_{i}^{\dagger}+\frac{\lambda \mathrm{e}^{i \vec{Q} \vec{R}_{i}}}{\sqrt{1+\mu^{2}}}\left(t_{i x}^{\dagger}+i \mu t_{i y}^{\dagger}\right)\right], \\
\tilde{t}_{i x}^{\dagger} & =\frac{1}{\sqrt{1+\lambda^{2}}}\left[-\lambda \mathrm{e}^{i \vec{Q} \vec{R}_{i}} s_{i}^{\dagger}+\frac{1}{\sqrt{1+\mu^{2}}}\left(t_{i x}^{\dagger}+i \mu t_{i y}^{\dagger}\right)\right], \\
\tilde{t}_{i y}^{\dagger} & =\frac{1}{\sqrt{1+\mu^{2}}}\left(t_{i y}^{\dagger}+i \mu t_{i x}^{\dagger}\right) \\
\tilde{t}_{i z}^{\dagger} & =t_{i z}^{\dagger},
\end{aligned}
$$

where $\lambda$ and $\mu$ are condensate amplitudes. The role of the singlet product state is now taken by $\left|\psi_{0}\right\rangle=\prod_{i} \tilde{s}_{i}^{\dagger}|0\rangle$. For $\lambda=\mu=0$ we have the original "paramagnetic" bond operators, while $\lambda=1, \mu=0$ describes a classical Néel state $\left|\psi_{0}\right\rangle$ and its local excitations (note that we have chosen the direction of the staggered magnetization to be in $x$ direction). Finally, for $\mu=1$ and $\lambda \rightarrow \infty$ the product state is the fully polarized state with all spins in $z$ direction. Below, we will employ ordering wavevectors $\vec{Q}=(\pi, \pi, 0)$ or $(\pi, \pi, \pi)$, both describing states with ferromagnetic correlations between 2nd vertical neighbors and reflecting the $\mathrm{Z}_{2}$ degeneracy w.r.t. the relative orientation of neighboring layers.

The Hamiltonian can be re-written in terms of the $\left\{\tilde{s}, \tilde{t_{\alpha}}\right\}$ operators; the corresponding lengthy expressions can be found in Ref. 29 and will not be reproduced here. The condensate parameters $\lambda$ and $\mu$ are determined by minimizing $\left\langle\psi_{0}|H| \psi_{0}\right\rangle$; this can be shown to be equivalent to eliminating linear $\tilde{t}^{\dagger}$ terms in $\mathcal{H}$. Then, as in the paramagnetic case, the $\tilde{t}_{\alpha}^{\dagger}$ can be treated as excitations on top of a background state, and $\tilde{s}$ will be formally set to unity. Subsequently, the Hamiltonian admits a harmonic approximation, by only keeping the quadratic terms in $\tilde{t}_{\alpha}$. The resulting $\mathcal{H}_{2}$ is solved by a Bogoliubov transformation, leading to new quasiparticles $\tau_{\vec{q} \alpha}$. For $\lambda=1$ and $\mu=0, \mathcal{H}_{2}$ is equivalent to conventional linear spin-wave theory of a Néel-ordered antiferromagnet.

In summary, the modified bond-operator approach interpolates between the triplon description of the paramagnet, spin waves of the antiferromagnet, and the flippedspin quasiparticle physics of the field-polarized ferromagnet. Within the harmonic approximation, the phase transitions turn out to be of second order, and the ordered phases have the correct number of Goldstone modes. We also note that an extension beyond the harmonic level is not obvious: Taking into account a hardcore repulsion of the $\tilde{t}_{\alpha}$ as above leads to a violation of the Goldstone theorem. Therefore our quantitative calculations in the ordered phases below will be restricted to the harmonic level. This is expected to be a reasonable approximation away from the phase transitions, and is also qualitatively correct near phase transition above the upper-critical dimension. However, we cannot capture critical behavior beyond mean-field, including possible logarithmic corrections occuring at phase transitions being at the uppercritical dimension (like a BEC transition in 2d).

\section{Observables}

In the harmonic approximation, static observables like magnetizations are calculated as expectation values with the ground state of $\mathcal{H}_{2}$ which is the vacuum of the Bogoliubov-transformed operators $\tau_{\vec{k} \alpha}$.

It may be tempting to calculate e.g. the $T=0$ uniform magnetization from the field dependence of the groundstate energy, $M=-\partial E_{0} / \partial H$. However, this procedure is incorrect in magnetically ordered phases for the following reason: In an ordered phase, the condensate parameters and hence the basis vectors are in general fielddependent, i.e., the expansion is done around a fielddependent product state. This implies that the quadratic part of the Hamiltonian will be field-dependent. In other words, in the harmonic approximation $\mathcal{H}_{2}$ corresponds to a different Hamiltonian for each field. Then, $-\partial E_{0} / \partial H$ contains, apart from the magnetization, an additional 
contribution arising from the field dependence of $\mathcal{H}_{2}$. We note that $M=-\partial E_{0} / \partial H$ is sometimes used in spin-wave theories for canted antiferromagnets - there, it yields incorrect results as well. However, the deviations from $\left\langle S_{z}\right\rangle$ are often small.

Dynamic properties, like the cross section for inelastic neutron scattering, can be expressed in terms of the Green's functions of the triplon quasiparticles. Beyond the harmonic approximation, this route needs to be taken for static observables as well.

\section{E. Relation to single-boson description}

Let us close this section with comments on the relation between the advocated bond-boson approach and effective theories based on a single-boson description often used in the field-driven cases. ${ }^{35}$

It is apparent, that near $H_{c 1}$ only the $\tau_{+}$boson is low in energy (equivalent to the $\Phi=\phi_{x}+i \phi_{y}$ mode of Sec. III), hence $\tau_{+}$is the only required degree of freedom in a critical theory for the $H_{c 1}$ transition. There are, however, a few subtleties: (i) Scattering processes between lowenergy and high-energy bosons contribute to terms in the low-energy theory. This is in fact the case in the ideal bct lattice when $J_{z z}=0$ in the Hamiltonian. Then, the bare boson dispersion is independent of $q_{z}$ at wavevectors $\left(\pi, \pi, q_{z}\right)$, and interactions are required to lift this degeneracy. As scattering processes between $\tau_{+}$bosons conserve particle number and hence are absent at $T=0$, one may conclude that the dispersion remains flat. This is incorrect, because scattering processes with the highenergy particles $\tau_{-, 0}$ are no longer number-conserving, inducing a finite dispersion. (ii) To fully describe the condensate with canted order throughout the phase diagram, a single complex parameter is insufficient, as clearly seen from the full bond-operator description (where both $\lambda$ and $\mu$ are needed). Using a condensate of $\tau_{+}$only is appropriate in the limit $J^{\prime} \ll J$, but this cannot describe states close to a quasiclassical Néel state.

This concludes our description of the methods.

\section{CASE (A): EQUIVALENT LAYERS AND FULL FRUSTRATION}

This section discusses in detail the situation (A) with an ideal bct structure of dimers, while situation (B) a structure with inequivalent layers and two dimers per unit cell - will be studied in Sec. VI. For the purpose of comparison with experiments, most numerical results shown below are for parameter values of possible relevance to the material $\mathrm{BaCuSi}_{2} \mathrm{O}_{6}$.

The dimensional reduction observed in $\mathrm{BaCuSi}_{2} \mathrm{O}_{6}$, i.e., the $2 \mathrm{~d}$ value of the shift exponent describing the BEC phase boundary, $T_{c} \propto\left(H-H_{c 1}\right)^{\psi}$ with $\psi=1$, was originally discussed in terms of frustration only, i.e. scenario (A). ${ }^{18}$ While the neutron scattering results of

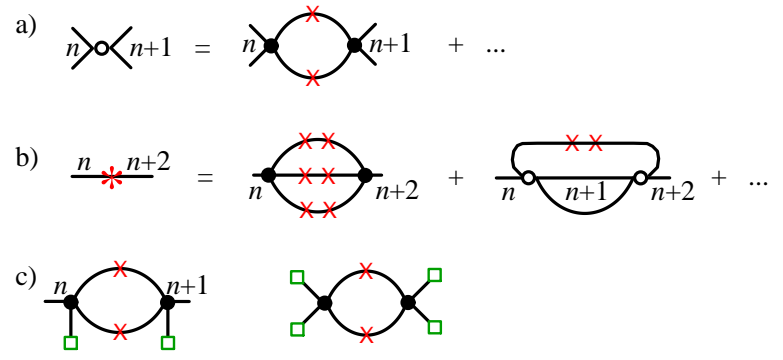

FIG. 3: Diagrams occurring in the perturbation expansion of the order-parameter theory $\mathcal{S}_{\phi}(7)$. The solid lines are $\phi$ propagators (depending on in-plane momentum $\vec{k}_{\|}$and layer index $n)$, the full circle is the local four-point vertex $\left(\propto u_{0}\right)$, the cross is the frustrated inter-layer hopping $\left(\propto \eta k_{x} k_{y}\right)$. a) Inter-layer density interaction [open circle, this includes both $u_{1}$ and $u_{2}$ terms in $\left.\mathcal{S}_{A B}(12)\right]$, generated from a $u_{0}^{2}$ process. For negative $u_{1}$, the $u_{1}\left(\vec{\phi}_{n} \cdot \vec{\phi}_{n+1}\right)^{2}$ term leads to collinear spin correlations in $z$ direction. b) Unfrustrated second-neighbor vertical hopping (star, $\propto \eta^{\prime}$ ), generated from interaction processes. This hopping is responsible for $3 \mathrm{~d}$ behavior at lowest energies, irrespective of the inter-layer frustration. c) Additional diagrams present in the ordered phase; the open square denotes the coupling to the condensate. The first diagram corresponds to nearest-neighbor vertical hopping, the second is the leading "vertical" contribution to the free energy.

Ref. 21 point to a somewhat different origin of quasi-2d behavior, namely scenario (B) with inequivalent dimers, the physics of the ideal bct structure is a very interesting and instructive situation to study. Some of the results described below were reported by us in Ref. 20 .

\section{A. Paramagnetic phase: Dispersions}

For one dimer per unit cell, there will be a single branch of magnetic triplet excitations in the zero-field singlet phase. Its in-plane dispersion caused by $J^{\prime}$ is unfrustrated, but the $c$ axis dispersion is heavily influenced by frustration: Away from the in-plane dispersion minimum at $\vec{q}_{\|}=(\pi, \pi)$ it is dominated by the frustrated hopping between adjacent layers, which leads to a (bare) dispersion of the form $J_{z} \cos \left(q_{x} / 2\right) \cos \left(q_{y} / 2\right) \cos q_{z}$. This vanishes at $\vec{q}_{\|}=(\pi, \pi)$, and only second-neighbor hopping contributes, with an additional dispersion $J_{z z} \cos 2 q_{z}$.

A key question, also relevant for the critical behavior, is the fate of the vertical dispersion in a model with bare $J_{z z}=0$. The arguments in Refs. 18 suggested that the dispersion would then be $q_{z}$-independent along $\left(\pi, \pi, q_{z}\right)$, rendering the critical behavior at $H_{c 1}$ truly two-dimensional. However, interaction processes invariably induce a symmetry-allowed effective vertical secondneighbor hopping ${ }^{20}$ of order $J_{z}^{4} / J^{3}$ - this effect is the one responsible for the absence of true dimensional reduction (with the exception of the high-field situation $H \geq H_{c 2}$, see Sec. V D 3 below).

Let us briefly repeat the perturbative arguments for 
a)
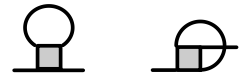

b)

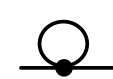

c)

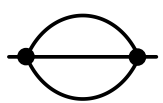

d)

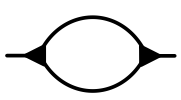

FIG. 4: Diagrams in the Brueckner bond-operator approach. The solid lines are $t_{\vec{q} \alpha}$ propagators, here depending on the full $3 \mathrm{~d}$ momentum $\vec{q}$. a) Self-energy from the hard-core repulsion; the shaded square is the effective four-point vertex $\Gamma$ (A5) obtained from a ladder summation. b) Leading self-energy from the quartic term $\mathcal{H}_{4}$, with the circle corresponding to $J_{4}$. c) Second-order self-energy in $\mathcal{H}_{4}$ - its $\mathcal{H}_{4 z}^{2}$ portion is required to obtain the leading term in the vertical dispersion on the ideal bct lattice. d) Second-order self-energy in $\mathcal{H}_{3 z}$, where the triangle is the three-point vertex of strength $J_{3}^{ \pm}$. The momentum space structure of the vertex suppresses this diagram at $\vec{q}_{\|}=(\pi, \pi)$.

the vertical dispersion. Within the effective orderparameter theory for $\vec{\phi}$, we need to look for processes which generate a contribution to the $\eta^{\prime}$ term in $\mathcal{S}_{A}$, Eq. (8), from the inter-layer interactions $\mathcal{S}_{A B}$, Eq. (12). The leading diagrams are in Fig. 3b, and are $\propto u_{0}^{2} \eta^{6}$ and $\propto u_{1,2}^{2} \eta^{2}$. The microscopic identification $\eta \propto J_{z}$, $u_{1,2} \propto J_{z}^{2}$ suggests that both diagrams are of order $J_{z}^{6}$. Higher-order processes will not change this result (except, perhaps, at a critical point, see Sec. VC below). However, the order parameter theory misses interactions between staggered and uniform magnetization fluctuations, as the latter are not contained in $\mathcal{S}_{\phi}$. This becomes clear when discussing the same physics in the bond-operator language. Relevant interaction terms are the hard-core term $\mathcal{H}_{U}(20)$ and the three- and four-point vertices arising from $J_{z}, \mathcal{H}_{3 z}(\mathrm{~A} 3)$, and $\mathcal{H}_{4 z}(\mathrm{~A} 1)$. (Note that the physics of $\mathcal{H}_{3 z}$ and $\mathcal{H}_{4 z}$ is absent from the effective orderparameter theory.) We will ignore effects of the in-plane part of $\mathcal{H}_{4}$ beyond the Hartree contribution, because the dominant overall renormalization arises from $\mathcal{H}_{U},{ }^{28}$ and qualitative changes from $\mathcal{H}_{4 \|}$ are not expected. Taking into account $\mathcal{H}_{U}$ yields a dispersion along $\left(\pi, \pi, q_{z}\right)$ proportional to $J_{z}^{6} \cos \left(2 q_{z}\right)$. This is easily understood, as the self-consistent Hartree diagram, Fig. 4a, contains the process in Fig. 3b2. Now consider the $\mathcal{H}_{4 z}^{2}$ portion of the diagram in Fig. 4c. Remarkably, it does generate a vertical dispersion proportional to $J_{z}^{4}$ (see App. A) hence this the leading contribution to the interactiongenerated vertical dispersion. We have therefore incorporated this diagram into the Brueckner approach. Finally, there is $\mathcal{H}_{3 z}$. Fig. $4 \mathrm{~d}$ shows the leading self-energy diagram, which, however, turns out to vanish at $\vec{q}_{\|}=(\pi, \pi)$. The contribution from $\mathcal{H}_{3 z}$ to the vertical dispersion is of order $J_{z}^{6}$; hence we will ignore $\mathcal{H}_{3 z}$ altogether.

A full result for the dispersion is shown in Fig. 5. The parameter values are chosen with an eye towards $\mathrm{BaCuSi}_{2} \mathrm{O}_{6}$, i.e., we have tried to match the in-plane

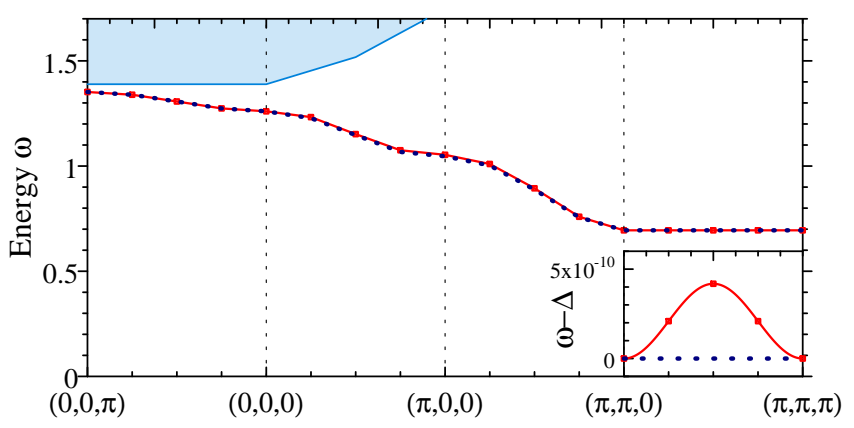

FIG. 5: Triplon dispersion (solid) and boundary of the twoparticle continuum (shaded), calculated using the Brueckner bond-operator approach (Sec. IV B and App. A) on an $8^{3}$ lattice. The parameter values are $J=1, J^{\prime} / J=0.15$, $-J_{2 z}=J_{4 z}=0.2 J^{\prime}, J_{3 z}=0, H=0$. The inset shows an energy zoom into the almost flat vertical dispersion. For comparison, the dotted line shows a dispersion calculated within the harmonic approximation, with parameter values chosen to match the Brueckner results at wavevectors $(0,0,0),(\pi, \pi, 0)$, and $(0,0, \pi): J=1.047, J^{\prime} / J=0.140,-J_{2 z} / J^{\prime}=0.196$.

dispersion averaged over the modes observed in Ref. 21, i.e., $J^{\prime} / J=0.15$. The vertical coupling is somewhat arbitrarily chosen as $-J_{2 z}=J_{4 z}=0.2 J^{\prime}$, giving a dispersion at the unfrustrated point, i.e., along $\left(0,0, q_{z}\right)$, of $\left(-0.096 J \cos q_{z}\right)$. The dispersion at the frustrated point is tiny, roughly $\left(-2 \cdot 10^{-10} J \cos 2 q_{z}\right)$, its bandwidth scales as $J_{z}^{4}$ as expected.

In Fig. 5 we illustrate that the in-plane dispersion obtained from the Brueckner approach can be reproduced using the harmonic bond-operator approximation describing non-interacting triplons with renormalized parameters. (Similar observations were made earlier e.g. in the context of spin ladders. ${ }^{36}$ ) The leading renormalization is in $J$ : triplon repulsion pushes the dispersion to higher energies, hence the "harmonic" $J$ is larger than the "true" (Brueckner) J. Of course, the renormalization in general depends on temperature, magnetic field and other parameters. Using the zero-field renormalized parameters gives a reasonable account of the field dependence of the spin gap, and overestimates $H_{c 1}$ only by a few percent (compared to the Brueckner approach). In the ordered phases and in the more complicated case of inequivalent layers (Sec. VI), where a Brueckner calculation is no longer feasible, we will exploit this fact, i.e., we will work with the harmonic approximation and parameter values chosen to match experimental data.

Moving closer to the zero-field transition by increasing $J^{\prime} / J$, the overall triplon bandwidth increases, see Fig. 6 . The interaction-generated bandwidth $E_{z}$ along $\left(\pi, \pi, q_{z}\right)$ strongly increases, as the relevant energy denominator in the fourth-order expression is given by the third power of an averaged triplon energy. The induced bandwidth will also depend on temperature, but this effect is exponentially suppressed for $T<\Delta$. From the numerical results, we are not able to track $E_{z}$ close to the phase transition, due to discretization errors on the finite lattice. Finally, 
upon applying a field, the triplon modes simply split as expected, Fig. 7.

After having established the vertical second-neighbor coupling, it is worth discussing the first-neighbor correlations. In the field-theory language, these arise from the $u_{1}$ term in $\mathcal{S}_{A B}(12)$ and are of orthogonal (collinear) type for $u_{1}>0\left(u_{1}<0\right)$, as the $u_{1}$ term is insensitive to the sign of $\vec{\phi}_{A, B}$. These correlations can be seen in fourpoint spin correlators only. The peculiar momentumspace structure of the $\eta$ term implies that two-point spin correlators between even and odd planes are strictly zero within the order-parameter theory in its paramagnetic phase. (The microscopic bond-operator approach shows weak ferromagnetic correlations between neighboring planes, which do not become long-ranged near the ordering transition.)

Microscopically, the $u_{1}$ term may originate from a biquadratic spin coupling as in $\mathcal{H}_{\text {coll }}$ (4). As above, one has to ask about the value of $u_{1}$ in the absence of a bare $J_{\text {coll }}$. In fact, $u_{1,2}$ will be interaction-generated as well, with the leading diagram $\propto J_{z}^{2}$ shown in Fig. 3a. Care needs to be taken when contracting two $u_{0}$ vertices because of the vector structure of the interactions. A straightforward analysis shows $u_{1}, u_{2}<0$ with $u_{2} / u_{1}=(N+4) / 4$, where $N$ is the number of order parameter components. $u_{1}<0$ means that collinear correlations are favored - this is consistent with the quasiclassical order-from-disorder mechanism operative inside the ordered phases, see Sec. VB. (Note that $u_{2}$ acquires additional contributions $\propto J_{z}$ near $H_{c 1,2}$ from a mean-field-like interaction of uniform magnetizations.)

\section{B. Antiferromagnetic phase: Order from disorder}

We now turn to the physics inside the antiferromagnetically ordered phases; the discussion of the phase transitions will be postponed to the following subsections.

As mentioned in the introduction, for classical moments on the bct lattice, antiferromagnetic layers are

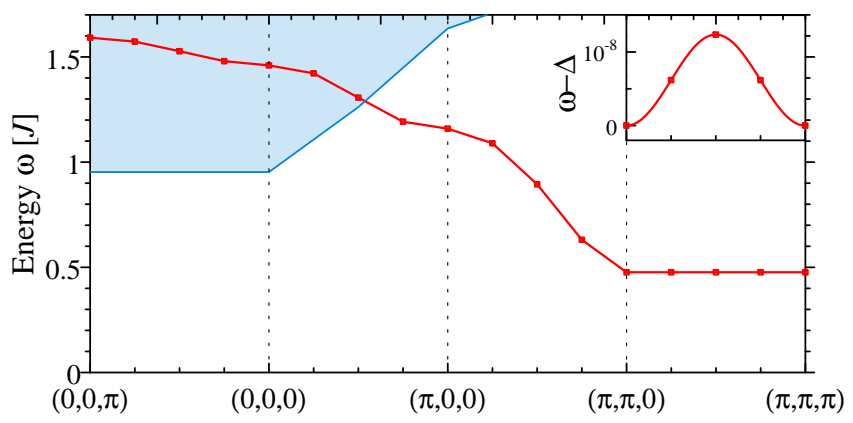

FIG. 6: Triplon dispersion (solid) and two-triplon continuum (shaded) as in Fig. 5, but with parameter values closer to the zero-field transition: $J=1, J^{\prime} / J=0.25,-J_{2 z}=J_{4 z}=0.2 J^{\prime}$, $J_{3 z}=0, H=0$. Compared to Fig. 5, the bandwidth along $\left(\pi, \pi, q_{z}\right)$ is significantly enhanced.

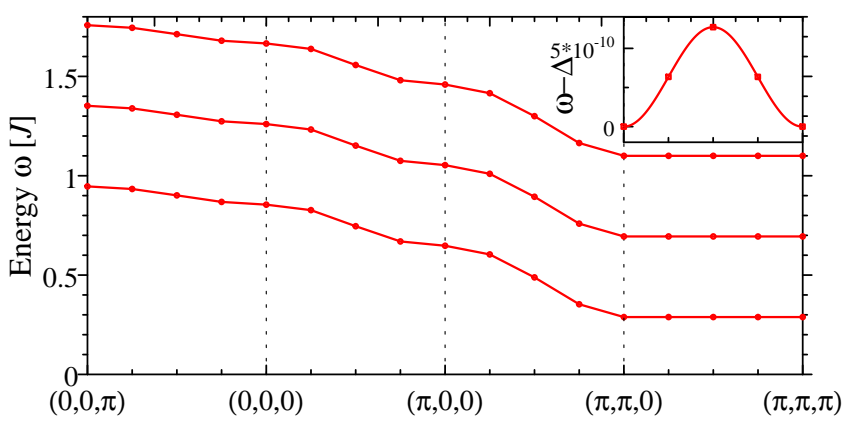

FIG. 7: Triplon dispersion as in Fig. 5, but now in a finite field: $J=1, J^{\prime} / J=0.15,-J_{2 z}=J_{4 z}=0.2 J^{\prime}, J_{3 z}=0$, $H=0.4$.

completely decoupled (provided $J_{z z}=0$ ).

\section{Spin-wave theory}

The effect of quantum fluctuations on the frustrated bct antiferromagnet has been initially studied using semiclassical spin-wave theory, ${ }^{39-41}$ and we briefly summarize the results here.

The simplest calculation, for a model with spins $S$ on sites of a bct lattice, assumes a spiral order with wavevector $\left(\pi, \pi, Q_{z}\right)$. In linear spin-wave approximation, the spin-wave spectrum is degenerate along the line $\left(\pi, \pi, q_{z}\right)$, signaling frustration. However, the ground state energy contains an inter-layer contribution of the form $\left(-J_{z}^{2} S / J^{\prime} \cos ^{2} Q_{z}\right)$, thus favoring collinear order with $Q_{z}=0$ or $Q_{z}=\pi$ - this term arises from the zeropoint energy of high-energy spin-wave modes. Including spin-wave interactions to order $1 / S$ removes the degeneracy in the dispersion along $\left(\pi, \pi, q_{z}\right)$, consistent with the assumed ordering wavevector - the dispersion is proportional to $\sqrt{S}\left|J_{z} \sin \left(q_{z} / 2\right)\right|$, i.e., corresponds to vertical first-neighbor hopping. ${ }^{40}$ Thus, this calculation predicts ferromagnetic order between second-neighbor planes, and collinear order between neighboring planes. A subsequent, more detailed, calculation ${ }^{41}$ relaxed the assumption of a single ordering wavevector and analyzed more general ordered states to high orders in $1 / S$. As a result, for $S=1 / 2$ a state with antiferromagnetic order between second-neighbor planes and collinear order between neighboring planes was found be selected by energy contributions of order $J_{z}^{4} /(J S)^{3}$ and $J_{z}^{6} /\left(J^{5} S\right)$. Thus, fully $3 \mathrm{~d}$ order is stabilized within the stacks of even and odd planes. The residual $\mathrm{Z}_{2}$ degeneracy specifying the relative orientation of the "even" and "odd" order parameter (Sec. III B) is left intact and hence spontaneously broken in the ordered state.

We note that the tendency towards collinear correlations can be mimicked ${ }^{24}$ by a biquadratic term in the Hamiltonian of the form (4), with $J_{\text {coll }} \propto-J_{z}^{2} / J^{\prime}-$ we will exploit this in the triplon bound-state calculation.

One may also consider order-from-disorder on the 
bct lattice in the different situation with non-vanishing second-neighbor vertical coupling $J_{z z}$. Then, 3d order in each of the tetragonal subsystems is already established at the classical level, but the two order parameters are decoupled. Quantum fluctuations are only needed to produce the collinear coupling $\left(\propto J_{z}^{2} / J^{\prime}\right)$, reducing the degeneracy to the residual $\mathrm{Z}_{2}$ symmetry. (This latter order-from-disorder mechanism is similar to the one in the much-studied $J_{1}-J_{2}$ Heisenberg model on the square lattice, in the limit of large $J_{2}$.)

\section{Field theory}

It is instructive to re-phrase the order-from-disorder mechanism in terms of the order-parameter field theory of Sec. III, with the relevant diagrams shown in Fig. 3. First, the magnetization orientations between adjacent layers are determined by the $u_{1}\left(\vec{\phi}_{n} \cdot \vec{\phi}_{n+1}\right)^{2}$ term. Indeed, we have argued above for $u_{1}<0$ which favors collinear correlations. Second, the type of $3 \mathrm{~d}$ ordering of secondneighbor layers is determined by the $\eta^{\prime} \vec{\phi}_{n} \cdot \vec{\phi}_{n+2}$ term. Third, the coupling to the condensate allows an effective nearest-neighbor hopping in $z$ direction, which was symmetry-forbidden in the paramagnetic phase. The diagrams, Fig. 3c, show that the order-from-disorder contributions to the vertical dispersion and to the free energy scale with the square and the fourth power of the order parameter, respectively.

\section{Bond operators}

In the Brueckner bond-operator approach, we can follow the properties in the paramagnetic phase up to the phase transition. From the single-particle dispersion, Fig. 5, we can read off $\eta^{\prime}$; we find $\eta^{\prime}<0$, i.e., a dispersion minimum at $(\pi, \pi, 0)$ and $(\pi, \pi, \pi)$. Triplon condensation at this wavevector implies ferromagnetic orientation between second-neighbor layers. This may appear inconsistent with the above-mentioned result of Ref. 41, but the latter strictly only applies in the semiclassical limit. The collinear inter-layer correlations are contained in four-point triplon correlators. In those cases where we find a triplon bound state (Sec. V C 2 below), its internal structure implies collinear correlations and its dispersion has a minimum at wavevector $(0,0,0)$. Condensation of this bound state implies uniform bond order (see Sec. III B) and again ferromagnetic orientation between second-neighbor layers. [The ordering pattern of Ref. 41 would correspond to condensation of $\Psi$ at $(0,0, \pi)$.]

Inside the ordered phases, our bond-operator calculations are restricted to the harmonic approximation. Here, the dispersion degeneracy along $\left(\pi, \pi, q_{z}\right)$ is not lifted: there are too many Goldstone modes. Nevertheless, we expect energy-integrated properties like magnetizations to be semi-quantitatively correct. Some results in the field-induced canted phase are shown in Fig. 9 below.

\section{Zero-field quantum phase transition}

The quantum paramagnet can be driven into an antiferromagnetically ordered state at zero field by increasing $J^{\prime} / J$. Experimentally, the application of pressure changes $J$ and $J^{\prime}$ via a modification of bond lengths and angles. The coupled-dimer material $\mathrm{TlCuCl}_{3}$ is driven into an ordered state upon application of pressure, hence $J^{\prime} / J$ is increased here; for $\mathrm{BaCuSi}_{2} \mathrm{O}_{6}$ systematic pressure studies have not been performed to our knowledge.

In an unfrustrated system, the zero-field ordering transition breaks the SU(2) spin symmetry and has dynamic exponent $z=1$. In a spatially anisotropic, i.e., layered, system with a $3 \mathrm{~d}$ scale $E_{z}$, the quantum critical behavior is $2 \mathrm{~d}$ for energies or temperatures above $E_{z}$ while it is 3 d below $E_{z}$. Importantly, $E_{z}$ is essentially given by the bandwidth of the vertical dispersion near the ordering wavevector.

For the frustrated bct system, several complications arise which we briefly address in the following: There is the additional $\mathrm{Z}_{2}$ symmetry to be broken, and the vertical dispersion consists of a bare frustrated part and an interaction-generated unfrustrated part.

\section{Interaction-generated dispersion}

If the bare $J_{z z}$ vanishes, then a vertical dispersion along $\left(\pi, \pi, q_{z}\right)$ (determining the $3 \mathrm{~d}$ crossover scale) only arises from interactions. The leading term is of second order in a four-point vertex (Sec. V A), it strongly depends on the distance to criticality because the spin gap enters the denominator of the perturbative expression.

The simplest perturbative estimate is reliable in the paramagnetic phase for not too large $J_{z}$, but may fail at criticality due to a non-trivial flow of the four-point vertex. This happens at or below the upper critical dimension $d_{c}^{+}$and is signaled by infrared divergencies in the next-order diagrams. The renormalization group thus has a rather interesting structure (assuming a vanishing bare $\eta^{\prime}$ ): one-loop accuracy is sufficient to study the flow of the four-point vertex, but the strongly relevant two-point vertex $\eta^{\prime}$ is only generated at two loops. (A similar situation has recently been studied in the context of spin chains with a frustrated inter-chain coupling. ${ }^{42}$ ) Here we shall not perform a consistent two-loop renormalization group treatment; we expect that the scaling $E_{z} \propto J_{z}^{4}$ continues to hold for small $J_{z}$, albeit with nontrivial finite-temperature corrections to the prefactor in the quantum critical regime. (The $J_{z}^{4}$ scale is expected to evolve continuously into the $J_{z}^{4}$ scale of the ordered phase, Sec. VB, which decides between ferro- and antiferromagnetic ordering between second-neighbor layers.)

We also note that the bond-operator calculation partially accounts for the above renormalization effects due to the self-consistent resummation of vertex diagrams. (For the field-driven case, such a strong renormalization of $\eta^{\prime}$ does not arise, as the interaction processes nec- 
essarily involve the high-energy triplon branches, due to particle-number conservation within the low-energy branch.)

\section{Bound state condensation and split transition}

As discussed in Sec. III B, the $\mathrm{Z}_{2}$ symmetry can be broken via condensation of the bond order parameter $\Psi$ before magnetic order occurs. $\Psi$ corresponds to a singlet bound state of two triplons. ${ }^{37}$ While the transition to bond order may be studied using a mean-field approach, the bound-state dynamics requires more effort. As in Ref. 37, we have solved a Bethe-Salpeter equation for two-triplon bound states. The required input encompasses the single-triplon dispersion - to be taken from the Brueckner bond-operator calculation - and the triplon four-point scattering vertex. As self-consistency in the two-particle sector is beyond reach, it is reasonable to work with a bare scattering vertex, with contributions from $\mathcal{H}_{4}$ and $\mathcal{H}_{U}$. However, from our analysis it is clear that the effective biquadratic inter-layer interaction, represented by $u_{1}\left(\vec{\phi}_{n} \cdot \vec{\phi}_{n+1}\right)^{2}$ in the field theory, is relevant for triplon attraction. We therefore include by hand $\mathcal{H}_{\text {coll }}$ (4) which mimicks the effect of the collinear interaction; details are relegated to App. B.

Interestingly, for triplon attraction the couplings $\left(-J_{\text {coll }}\right)$ and $\left(+J_{4 z}\right)$ act in a very similar way: both can cause inter-layer binding. While the triplon binding effect of $\mathcal{H}_{\text {coll }}$ is easily understood in the order-parameter language, this is more subtle with $\mathcal{H}_{4 z}$. First, we observe that the relevant part of $\mathcal{H}_{4 z}$ involves the $u n i$ form (as opposed to staggered) degrees of freedom of the dimers. Second, $\Psi$ condensation represents bond order arising from quantum-mechanical singlet formation, which is common for frustrated spin- $1 / 2$ systems. ${ }^{38}$ (Note that the attractive force is linear in $J_{4 z}$.)

In zero field, we find a singlet bound state below the two-particle continuum only for sufficiently strong attraction $\left(J_{4 z}-J_{\text {coll }}\right)$. This is plausible, as small attraction in $2 \mathrm{~d}$ causes only an exponentially shallow bound state, while in $3 \mathrm{~d}$ a finite attraction is needed for binding this simply follows from the properties of one particle with quadratic dispersion moving in a potential well. The wavefunction of the lowest bound state changes sign under 90-degree in-plane rotations of the internal coordinate; from the symmetry considerations in Sec. III we conclude that this bound state corresponds to a $\Psi$ quantum as anticipated. The bound-state dispersion has its minimum at $(0,0,0)$ and a bandwidth along $\left(0,0, q_{z}\right)$ of $J_{z}^{4}$, inherited from the two-triplon continuum. A condensation of this singlet bound state corresponds to the $\mathrm{Z}_{2}$ symmetry breaking advocated in Sec. III B. The bound state is unaffected by a Zeeman field, hence for fields larger than the triplon binding energy the bound state ceases to exist below the two-particle gap.

In conclusion, both of the following scenarios may be realized (Fig. 8): (i) Two transitions: Upon increasing a)

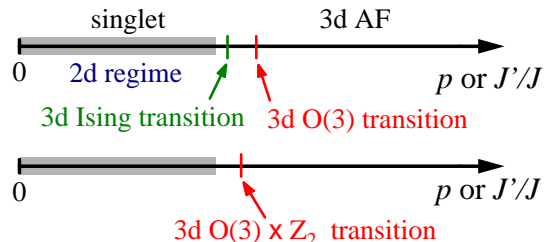

b)

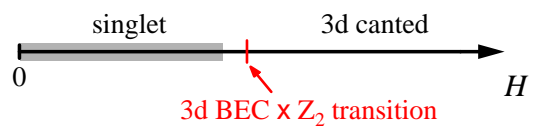

FIG. 8: Schematic $T=0$ phase diagrams for the bct lattice coupled-dimer model, for the situation of equivalent layers with perfect frustration. a) Pressure tuning - here generically two scenarios are possible (where the second one applies for the parameters relevant to $\left.\mathrm{BaCuSi}_{2} \mathrm{O}_{6}\right)$. b) Field tuning.

$J^{\prime} / J$, there is first a quantum Ising transition with $z=1$ where bond order is established through condensation of $\Psi$. This transition is asymptotically $3 \mathrm{~d}$ : the vertical $\Psi$ dispersion is of order $J_{z}^{4}$ along $\left(0,0, q_{z}\right)$. Subsequently, magnetic order will be established in a second transition where $\vec{\phi}$ condenses and the $\mathrm{SU}(2)$ symmetry is broken. The second transition has $z=1$ and is of conventional $\mathrm{O}(3)$ type, in the sense that frustration is removed by the $\Psi$ condensate, i.e., by the term $\lambda\langle\Psi\rangle \vec{\phi}_{n} \cdot \vec{\phi}_{n+1}$ [see Eq. (14)]. The distance of the transitions is determined by the triplon binding energy. (ii) One transition: the condensation of $\vec{\phi}$ breaks the $\mathrm{SU}(2) \times \mathrm{Z}_{2}$ symmetry in one step. This transition is again asymptotically $3 \mathrm{~d}$; a detailed study of the critical behavior shall not be undertaken here.

For the parameter values of possible relevance to $\mathrm{BaCuSi}_{2} \mathrm{O}_{6}$, we see no indication for bound states; hence case (ii) applies. Unfortunately, the numerical BetheSalpeter calculation suffers from severe finite-size effects, therefore we cannot reliably resolve small binding energies. We also note that the inclusion of lattice effects can modify the behavior: for instance, bond order could occur far before magnetic order sets in - this applies not only to $T=0$ but also to the finite- $T$ transitions.

\section{Anisotropies and further perturbations}

Perturbations beyond the model Hamiltonian (1) can modify the asymptotic critical behavior: Those include magnetic anisotropies of Dshyaloshinski-Moriya or dipolar type, as well as coupling to nuclear spins. In particular, anisotropy terms are relevant perturbations to the $\mathrm{O}(3)$ critical points, rendering the asymptotic critical behavior Ising-like. 

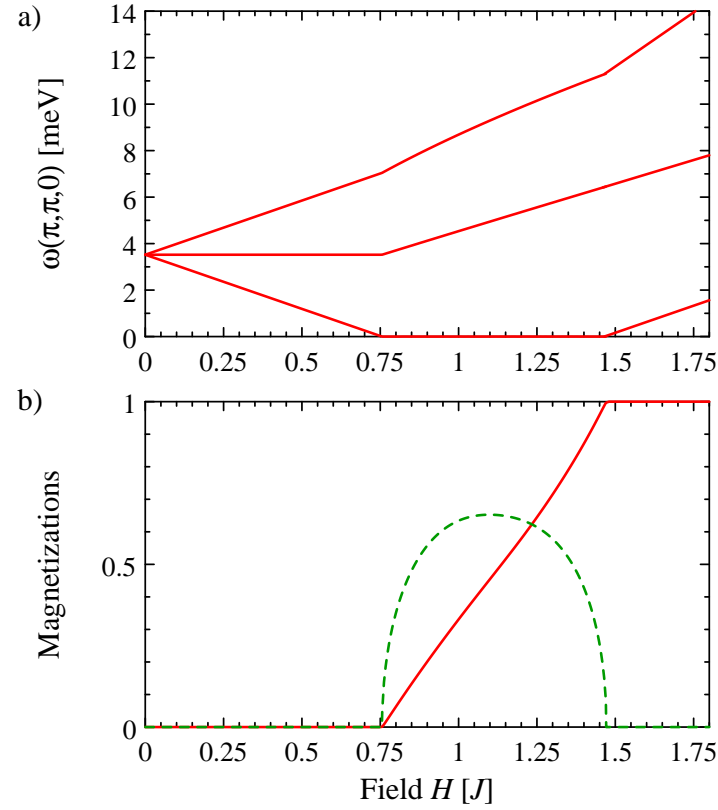

FIG. 9: a) Triplon energy gaps and b) uniform (solid) as well as staggered (dashed) magnetization, calculated as function of the external field $H$ using bond operators in the harmonic approximation. Parameter values describe the ideal bct lattice: $J=4.66 \mathrm{meV}, J^{\prime}=0.5 \mathrm{meV},-J_{2 z}=0.1 \mathrm{meV}$.

\section{Field-driven quantum phase transitions}

\section{Lower critical field $H_{c 1}$}

The ground state of the quantum paramagnet is unaffected by applying a Zeeman field, however, the triplon excitations will split, leading to a spin gap decreasing with applied field. At at critical field $H_{c 1}$, given by the zero-field gap, the lowest triplon mode becomes soft, and antiferromagnetic order perpendicular to the field is established. The phase transition is in the dilute Bose gas universality class, with dynamical exponent $z=2$, and breaks a U(1) symmetry. ${ }^{4}$

As above, the energy scale $E_{z}$ for the dimensional crossover between $2 \mathrm{~d}$ and $3 \mathrm{~d}$ is given by the vertical bandwidth of the single-particle dispersion near the minimum wavevector. In bct lattice coupled-dimer model with perfect inter-layer frustration, this scale is again given by the sum of the bare vertical second-neighbor hopping, $J_{z z}$, and the corresponding interaction-induced contribution - for the latter the perturbative estimate $J_{z}^{4} / J^{3}$ is now reliable even at the transition, because the relevant $T=0$ interaction processes involve high-energy triplons. At the transition, $\mathrm{U}(1)$ and Ising symmetries are broken simultaneously, Fig. 8b, as singlet bound states are irrelevant in finite field. The phase transition is still of the dilute Bose gas type, i.e. with $z=2$ and mean-field exponents, supplemented by logarithmic corrections in the $2 \mathrm{~d}$ regime. (Magnetic anisotropies beyond the model
(1) can modify the critical behavior at lowest energies as noted above; a Dshyaloshinski-Moriya interaction may even smear out the phase transition.)

For numerical calculations we resort to the harmonic bond-operator method. To this end, we employ parameters $J=4.66 \mathrm{meV}, J^{\prime}=0.5 \mathrm{meV},-J_{2 z}=0.1 \mathrm{meV}$, which roughly reproduce (within the harmonic approximation) the $\mathrm{BaCuSi}_{2} \mathrm{O}_{6}$ mode dispersions (averaged over the multiple modes). Results for mode gaps and magnetizations are shown in Fig. 9 (here $\vec{Q}=(\pi, \pi, 0)$ ).

\section{Phase boundary: $3 d$ critical behavior at elevated temperature?}

While inter-layer frustration leads to well-defined $2 \mathrm{~d}$ quantum critical behavior at intermediate energies or temperatures, one can ask whether $3 \mathrm{~d}$ critical behavior (with associated exponents) is restored at higher energies - this was proposed on the basis of the experiments on $\mathrm{BaCuSi}_{2} \mathrm{O}_{6}$ in Ref. 16.

While there is a sizeable vertical dispersion of the magnetic modes away from in-plane wavevector $(\pi, \pi)$, which may be associated with $3 \mathrm{~d}$ behavior, the thermodynamics is (to leading order) determined by the density of states of all magnetic modes. Here the alternating sign of the inter-layer hopping (as function of in-plane momentum) turns out to be crucial, because the $3 \mathrm{~d}$-like contributions to the density of states tend to cancel. As a result, the density of states does never cross over to the power law characteristic for $3 \mathrm{~d}$ - this applies to both zero-field and finite-field cases.

Turning to the location of the boundary of the ordered phase: this can be estimated within the order-parameter field theory by calculating the finite-temperature mass correction, i.e., the temperature dependence of the order parameter self-energy, on the paramagnetic side. For a transition above the upper critical dimension, the lowestorder estimate given by the Hartree diagram is usually sufficient; what enters the Hartree diagram is precisely the density of states of the magnetic modes. Performing the integral numerically nicely shows a crossover from $\Sigma(T)-\Sigma(0) \propto T^{3 / 2}$ for $T$ below the tiny $E_{z}$ to $\Sigma(T)-$ $\Sigma(0) \propto T$ above $E_{z}$, but no further crossover to another well-defined power law.

This strongly suggests that experimentally observed deviations from the $2 \mathrm{~d}$ critical power laws (above $1 \mathrm{~K}$ ) have nothing to do with $3 \mathrm{~d}$ critical behavior, but instead indicate that one leaves the critical regime (in the sense that the correlation length is no longer large, or that the density of triplons in the ground state is no longer dilute).

\section{Upper critical field $H_{c 2}$}

For large external fields, $H>H_{c 2}$, the ground state of the coupled-dimer model is fully polarized, and a quantum phase transition to a canted state occurs at $H_{c 2}$ 
which is in the dilute Bose gas universality class as well.

In contrast to the paramagnetic low-field phase, the wavefunctions for the ground state and the one-particle excitations above $H_{c 2}$ are exactly known: A Bloch wave of one spin flip on top of the ferromagnetic background is an exact eigenstate of $\mathcal{H}$. This has interesting consequences: although these spin-flip particles have a hardcore interaction, scattering processes are rare at low $T$, as the equilibrium particle density vanishes as $T \rightarrow 0$. Thus, the interaction corrections to the bare dispersion are exponentially suppressed above $H_{c 2} \cdot{ }^{19}$

Hence, in a model without bare $J_{z z}$, the vertical mode dispersion vanishes identically at $T=0$ for $H>H_{c 2}$, and will only be induced by thermal processes in the quantum critical regime. This suggests that the asymptotic critical behavior is two-dimensional. ${ }^{19}$

\section{E. Classical phase transition and Néel temperature}

Given the order-from-disorder mechanism, which generates an effective $3 \mathrm{~d}$ coupling proportional to the square of the order parameter itself, and given the MerminWagner theorem, the obvious question about the nature of the finite-temperature transition arises. The issue appears particularly relevant for $\mathrm{O}(3)$ symmetry, i.e., in the zero-field case: Starting in the ordered phase at low $T$, the system has a robust (effective) vertical coupling of order $J_{z}$, and one would hence predict a Néel temperature $T_{\mathrm{N}}$ roughly given by $J^{\prime} / \ln \left(J^{\prime} / J_{z}\right)$ (in the limit of small $J_{z}$, and $J^{\prime}$ is the relevant in-plane coupling constant). ${ }^{43}$ However, in the high-temperature paramagnetic phase the vertical coupling is tiny, i.e., only given by the effective $J_{z z} \propto J_{z}^{4}$. Upon cooling from high $T$ this results in an instability of the paramagnetic phase at a temperature which is smaller by a factor of 4 compared to the above estimate. This argument indicates that the finite-temperature transition could be discontinuous, but precursor effects of the order-from-disorder mechanism may counteract, rendering the transition continuous. A reliable self-consistent treatment of this problem appears difficult and is beyond the scope of the paper.

In any case, it is clear that the Néel temperature will strongly depend on the frustrated inter-layer coupling $J_{z}$. This applies not only to the situation with $\mathrm{O}(3)$ symmetry, but also to the $\mathrm{O}(2)$ case: here, the ordering temperature without vertical coupling does not vanish, but is given by the Kosterlitz-Thouless temperature $T_{\mathrm{KT}}$, and $J_{z}$ leads to a logarithmic enhancement of $T_{\mathrm{N}}$ compared to $T_{\mathrm{KT}}$. If the inter-layer coupling is the sum of an unfrustrated and a frustrated part, then both will contribute to $T_{\mathrm{N}}$ - this is likely the scenario relevant to $\mathrm{BaCuSi}_{2} \mathrm{O}_{6}$.

\section{CASE (B): INEQUIVALENT LAYERS}

We now turn to the case where the ideal bct structure is distorted such that the unit cell is enlarged to contain multiple dimers. This modification is suggested by the results of zero-field inelastic neutron scattering on $\mathrm{BaCuSi}_{2} \mathrm{O}_{6}$, where indications for at least two modes were found. ${ }^{21}$

As discussed in Sec. II B, we restrict our calculations to a situation with two types of inequivalent layers, $A$ and $B$, which are stacked in an alternating fashion. (More complicated symmetry-breaking patterns only lead to quantitative modifications.) We will consider both the cases of perfect and imperfect frustration, the latter modeled by unequal vertical couplings along the two diagonals, see Sec. II B.

The different microscopic couplings $J_{A, B}$ and $J_{A, B}^{\prime}$ in the even and odd layers then translate into unequal order parameter masses $m_{\phi A, B}$ and velocities $c_{A, B}$ in Eq. (8), whereas imperfect frustration implies finite $\kappa$ in Eq. (12). (In the following we will assume $m_{\phi A}<m_{\phi B}$.)

Numerical results will be obtained from the bondoperator theory in the harmonic approximation, applied to both the disordered and ordered phases. For simplicity, we will not take into account any effects beyond quadratic terms; this implies that we miss, e.g., effects of the collinear coupling between the two condensates. This will affect some low-energy properties and will be noted below, but the gross features of the results can be expected to be correct.

\section{A. Paramagnetic phase: Dispersions and reduced dimensionality}

In the situation of two positive triplon masses, the thermodynamic and magnetic properties of the system will be governed by the smaller of the two gaps. The primary signature of the doubled unit cell is the presence of two triplet modes at fixed wavevector (instead of one). Let us focus on the dispersion of these modes, in a regime of small to moderate vertical couplings $J_{z}$ (neglecting the tiny $\left.J_{z z}\right)$. Then, the in-plane dispersion is conventional and dominated by the $J, J^{\prime}$ values. At fixed $\vec{q}_{\|}$, the energy of the two modes differs by $\Delta \omega_{q}$. For $J_{z} \ll \Delta \omega_{q}$, the vertical dispersion arises only in second order in $J_{z}$ (!), i.e., $\propto J_{z}^{2} \cos \left(2 q_{z}\right) / \Delta \omega_{q}$. In contrast, for $J_{z} \gg \Delta \omega_{q}$ (which includes the case of equivalent planes) the vertical dispersion is $\propto J_{z}$ as usual.

Hence, inequivalent layers present an alternative mechanism for dimensional reduction, which moreover is effective for all in-plane wavevectors and in the absence of perfect frustration. Explicitly: for imperfect frustration, the relevant $3 \mathrm{~d}$ energy scale [being the bandwidth along $\left.\left(\pi, \pi, q_{z}\right)\right]$ is now $\propto J_{z}^{2}$ for small $J_{z}$. Note that perfect frustration results in a prefactor of $\cos ^{2}\left(q_{x} / 2\right) \cos ^{2}\left(q_{y} / 2\right)$ in front of the bare vertical hopping, which leaves us with the interaction-induced contribution to the vertical coupling at $q_{x}=\pi$ or $q_{y}=\pi$, which is still of order $J_{z}^{4}$.

Let us make these statements quantitative, by fitting the $\mathrm{BaCuSi}_{2} \mathrm{O}_{6}$ neutron scattering data of Ruegg et al. ${ }^{21}$. As we model only two inequivalent planes, we take the 


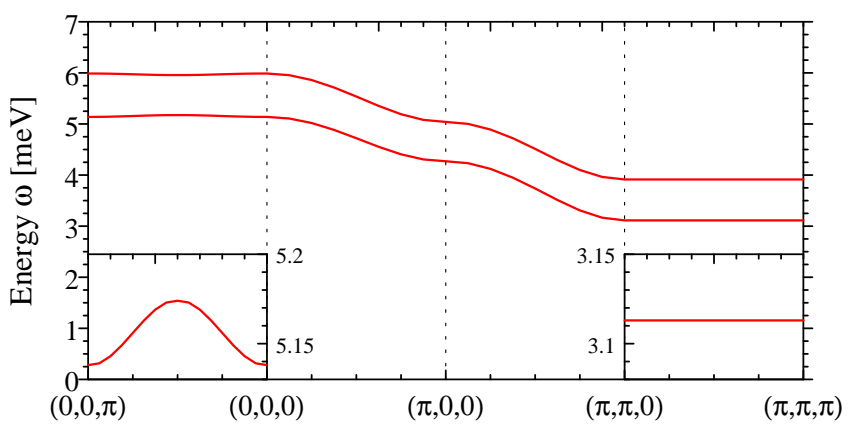

FIG. 10: Zero-field triplon dispersion for inequivalent layers with perfect frustration, calculated using the harmonic bondoperator approximation. Parameter values are: $J_{A}=4.27$ $\mathrm{meV}, J_{B}=5.04 \mathrm{meV}, J_{A}^{\prime}=J_{B}^{\prime}=0.5 \mathrm{meV},-J_{2 z}=J_{z}=$ $0.1 \mathrm{meV}$. Compared to the equivalent-layer case, the vertical dispersion at the unfrustrated point, i.e., along $\left(0,0, q_{z}\right)$, is both quenched and modified in shape, from $\propto J_{z} \cos q_{z}$ to $\propto \pm J_{z}^{2} \cos 2 q_{z}$.

neutron peaks with smallest and largest energies and find the (effective) couplings $J_{A}=4.27 \mathrm{meV}, J_{B}=5.04 \mathrm{meV}$, $J_{A}^{\prime}=J_{B}^{\prime}=0.5 \mathrm{meV}$, being essentially identical to the ones estimated in Ref. 21. Not much is known about the values of the vertical couplings in $\mathrm{BaCuSi}_{2} \mathrm{O}_{6}$. The simplest assumption is perfect frustration - a sample result for the zero-field triplon dispersion for $J_{z}=0.1 \mathrm{meV}$ is shown in Fig. 10. The vertical bandwidth along $\left(0,0, q_{z}\right)$ is roughly $0.04 \mathrm{meV}$ : compared to the equivalent-layer case, it is suppressed here by a factor of 5 , and it follows $\cos 2 q_{z}$ instead of $\cos q_{z}$ (!). The bandwidth along $\left(\pi, \pi, q_{z}\right)$ vanishes due to the perfect frustration. (The tiny $J_{z}^{4}$ contribution is not captured in the harmonic approximation.) For comparison, in Fig. 11 we also show dispersions in a situation with imperfect frustration, with couplings along the two inequivalent diagonals of strength $J_{z 1}=0.15 \mathrm{meV}, J_{z 2}=0.05 \mathrm{meV}$. Here, the bandwidth at the unfrustrated point is as before, but along $\left(\pi, \pi, q_{z}\right)$ we now observe a dispersion of width $0.025 \mathrm{meV}$.

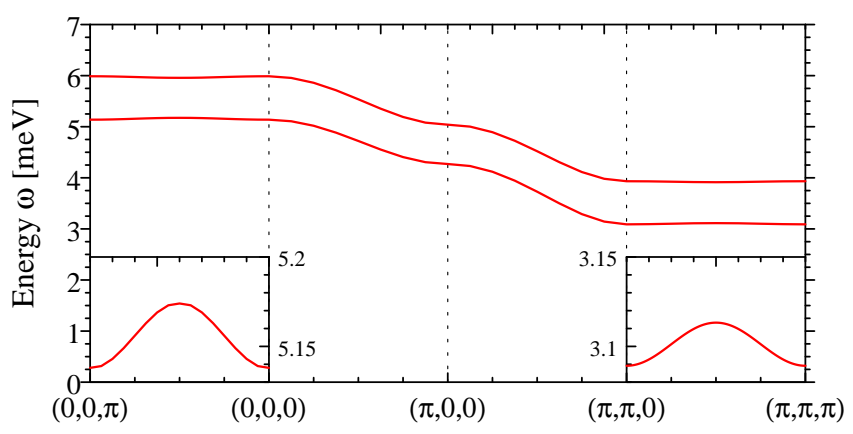

FIG. 11: As in Fig. 10, but now for inequivalent layers with imperfect frustration. Parameter values are: $J_{A}=4.27 \mathrm{meV}$, $J_{B}=5.04 \mathrm{meV}, J_{A}^{\prime}=J_{B}^{\prime}=0.5 \mathrm{meV}, J_{z 1}=0.05 \mathrm{meV}$, $J_{z 2}=0.15 \mathrm{meV}$ - the latter two values are the couplings along the two inequivalent $J_{z}$ diagonals, see Fig. $1 \mathrm{~b}$.
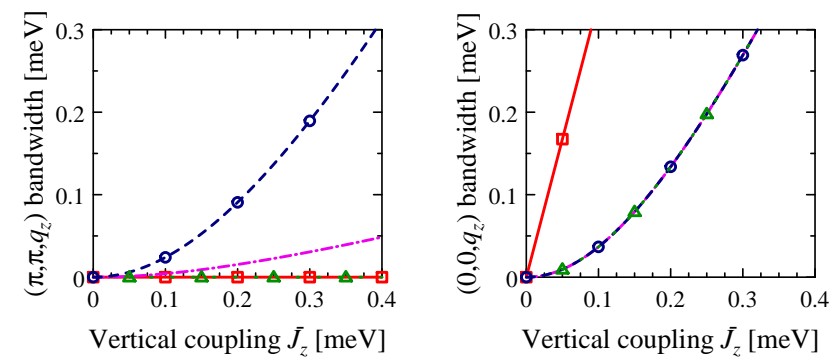

FIG. 12: Bandwidths of the vertical dispersion along $\left(\pi, \pi, q_{z}\right)$ and $\left(0,0, q_{z}\right)$ as function of the average $\bar{J}_{z}$, for equivalent layers $(J=4.66 \mathrm{meV})$ and perfect frustration (solid, squares), inequivalent layers $\left(J_{A}=4.27 \mathrm{meV}, J_{B}=5.04 \mathrm{meV}\right)$ with perfect frustration (dotted, triangles), imperfect frustration with $J_{z 1} / J_{z 2}=3$ (dashed, circles), and imperfect frustration plus vertical modulation with $J_{z A 1} / J_{z A 2}=11 / 9, J_{z B 1} / J_{z B 2}=19$, $\bar{J}_{z A}=\bar{J}_{z B}$ (dash-dot, no symbols, see also Fig. 18) below. In all cases, $J^{\prime}=0.5 \mathrm{meV}$.

In Figs. 12,13 we show vertical bandwidths as function of $J_{z}$ for the above values of in-plane coupling. For $J_{z} \ll \Delta \omega_{q}$, these bandwidths scale as $\left(J_{z A 1}-\right.$ $\left.J_{z A 2}\right)\left(J_{z B 1}-J_{z B 2}\right) / \Delta \omega_{q}$ at the frustrated point and $\left(J_{z A 1}+J_{z A 2}\right)\left(J_{z B 1}+J_{z B 2}\right) / \Delta \omega_{q}$ at the unfrustrated point [with the conventions of Eq. (6)]. In Fig. 13 the vertical bandwidths are shown as function of the two-dimensional in-plane momentum, for the parameter values of Figs. 10 and 18 (below, this case features inequivalent vertical diagonals).

\section{B. Antiferromagnetic phases: Frustrated proximity effect}

The antiferromagnetic phases in the inequivalent-layer case, both at zero and finite field, can be nicely discussed using the order-parameter field theory. Due to the unequal masses for the $A$ and $B$ subsystems, a situation with $m_{\phi A}<0, m_{\phi B}>0$ can occur. Then, order in the

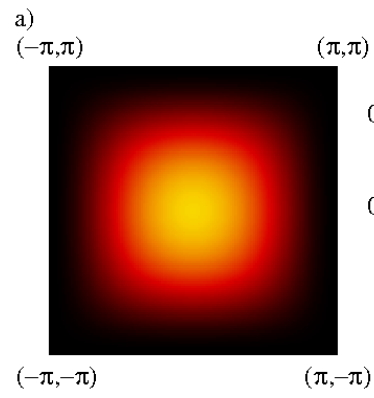

b)

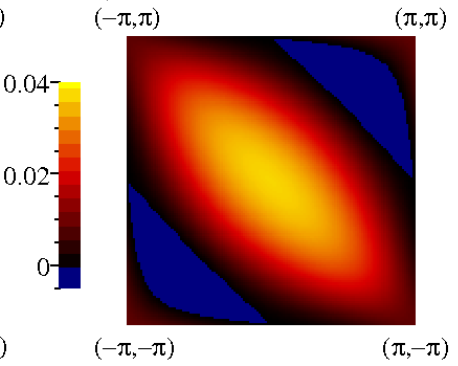

FIG. 13: Vertical dispersion of the lowest mode, $\omega\left(q_{x}, q_{y}, \pi / 2\right)-\omega\left(q_{x}, q_{y}, 0\right)$, as a function of in-plane momentum $\vec{q}_{\|}$for inequivalent-layer situations. a) Perfect frustration, parameter values as in Fig. 10. b) Imperfect frustration plus vertical modulation, parameter values as in Fig. 18) below. 
a)

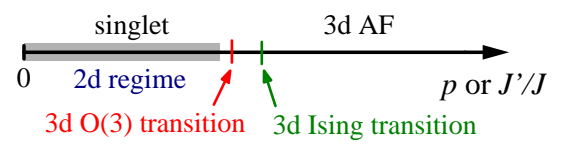

b)

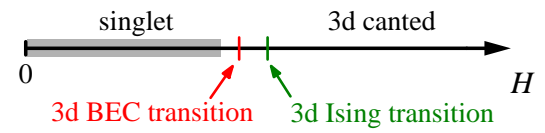

FIG. 14: Schematic $T=0$ phase diagrams for the coupleddimer model with inequivalent layers and perfect frustration. Due to the absence of a proximity effect, there is no coupling between the condensates on the subsystems $A$ and $B$ (on even and odd layers), leading to two magnetic transitions. a) Pressure tuning. b) Field tuning.

$A$ subsystem is established.

Usually, one would expect that $A$ order induces order in the $B$ subsystem as well, due to a proximity effect. Technically, a proximity effect arises from a linear coupling between $\vec{\phi}_{A}$ and $\vec{\phi}_{B}$ [the $\kappa$ term in $\mathcal{S}_{\phi A B}(12)$ ]. However, as detailed in Sec. III, this term is forbidden in the presence of perfect frustration, and hence the proximity effect is absent due to frustration! The only effect of the $A$ condensate on the $\vec{\phi}_{B}$ fluctuations is via the quartic terms which cause an anisotropy, see below.

The absence of a proximity effect also implies that the (strong) order-from-disorder mechanism discussed in Sec. VB is only present in phases with order in both $A$ and $B$ subsystems.

\section{Phase diagrams}

Given the absence of a proximity effect in the perfectly frustrated case, two distinct phase transitions will occur for the $A$ and $B$ subsystems. Depending on the relation between the parameters $J_{A, B}, J_{A, B}^{\prime}$, different phase diagrams may be realized, see Figs. 14,15.

There will be primary transitions between a disordered and a partially symmetry-broken phase, and secondary transitions between partially and fully symmetry-broken phases. Both types transitions extend to finite temperatures and will be visible as singularities in thermodynamic quantities. Breaking the frustration will turn the secondary transitions into crossovers, see Sec. VIE for explicit numerical results.

We believe that the presence or absence of these secondary transitions allows a clear-cut experimental distinction between perfect and imperfect frustration in the situation (B) of inequivalent layers.

\section{Zero-field phase transitions}

For perfect frustration, upon increasing the ratios $J^{\prime} / J$ (e.g. by applying pressure) either $\vec{\phi}_{A}$ or $\Psi$ may condense first. If the difference between the masses, $m_{\phi B}-m_{\phi A}$, is larger than binding energy of two $A$ and $B$ triplons (which is set by $J_{4 z}$ ), then the first transition is the magnetic ordering of $\vec{\phi}_{A}$. This transition is in the standard $\mathrm{O}(3)$ universality class and has $z=1$. The dimensional crossover between $3 \mathrm{~d}$ and $2 \mathrm{~d}$ critical behavior is set by $\eta_{A}^{\prime} \sim J_{z}^{4}$, as discussed in Sec. VC.

Ordering of $A$ introduces a mass anisotropy into the action for $\vec{\phi}_{B}$ through the term $u_{1}\left(\left\langle\vec{\phi}_{A}\right\rangle \cdot \vec{\phi}_{B}\right)^{2}$, which is of easy-plane (easy-axis) type for $u_{1}>0\left(u_{1}<0\right)$. Then, further increasing $J^{\prime} / J$ will lead to ordering of $\vec{\phi}_{B}$, with a quantum phase transition in the $\mathrm{O}(2)$ (Ising) universality class with $z=1$. Recall that $u_{1}<0$ is the generic situation in our coupled-dimer model, leading to a secondary Ising transition. The crossovers near the second transition are somewhat more complicated: with increasing energy, we go from $3 \mathrm{~d}$ Ising to $2 \mathrm{~d}$ Ising to $2 \mathrm{~d}$ Heisenberg, with the two crossover scales set by $\eta_{B}^{\prime} \propto J_{z}^{4}$ and $u_{1}\left\langle\vec{\phi}_{A}\right\rangle^{2} \propto J_{z}^{2}$.

For imperfect frustration there is only one magnetic transition in the $\mathrm{O}(3)$ universality class, and the secondary transition is smeared out. The energy scale relevant to the dimensional crossover from $3 \mathrm{~d}$ to $2 \mathrm{~d}$ is now determined by the "bare" triplon dispersion near the minimum wavevector $(\pi, \pi, 0)$ (i.e. interaction effects are subleading). As shown in Sec. VI A, this scale is proportional to $J_{z}^{2}$ (i.e. much larger than in the perfectly frustrated case).

\section{E. Field-driven phase transitions}

Similar to the zero-field transition, the field-driven transitions at $H_{c 1}$ and $H_{c 2}$ are split in the presence of perfect frustration due to the absence of a proximity effect. The primary $A$ ordering transition is in the dilute Bose gas (i.e. BEC) universality class with $z=2$. At $H_{c 1}$, the dimensional crossover between $3 \mathrm{~d}$ and $2 \mathrm{~d}$ criti-

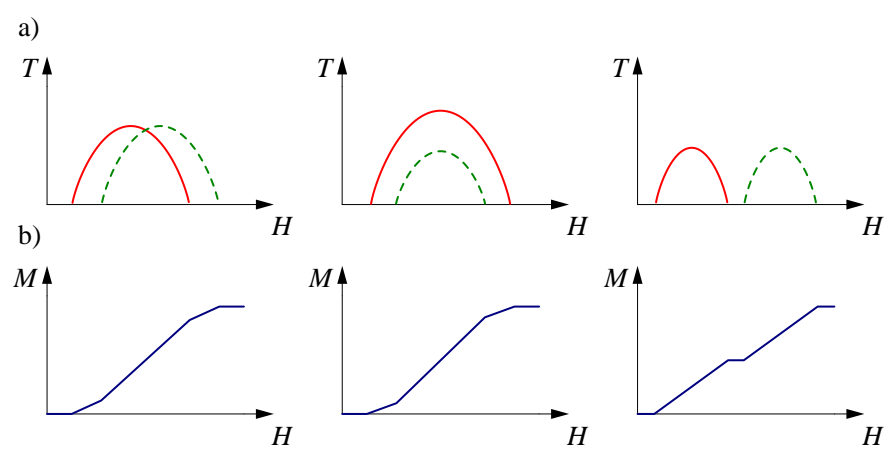

FIG. 15: a) Schematic overall temperature-field phase diagrams for the coupled-dimer model with inequivalent layers and perfect frustration, for different relations between $J_{A, B}$, $J_{A, B}^{\prime}$. Solid (dashed) lines show the phase transitions on the $A(B)$ subsystem. b) Schematic zero-temperature magnetization curves for the three scenarios in a). For perfect frustration, the slope will change discontinuously at the transitions. 

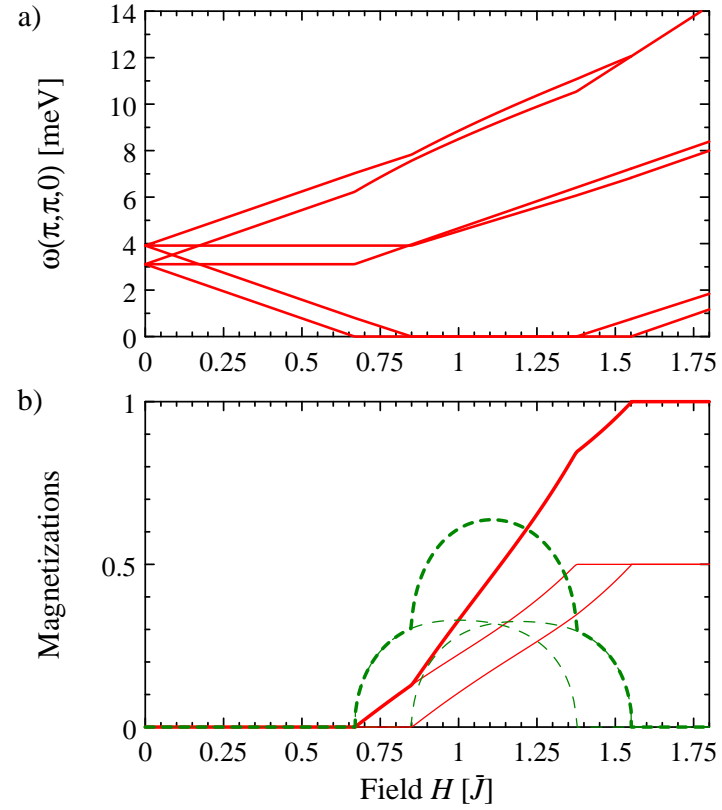

FIG. 16: a) Triplon energy gaps and b) uniform (solid) and staggered (dashed) magnetization as in Fig. 9, but for inequivalent layers with perfect frustration - the splitting of the phase transitions is clearly visible. Parameter values are: $J_{A}=4.27$ $\mathrm{meV}, J_{B}=5.04 \mathrm{meV}, J_{A}^{\prime}=J_{B}^{\prime}=0.5 \mathrm{meV},-J_{2 z}=J_{z}=0.1$ $\mathrm{meV}$. The field is displayed in units of the average $\bar{J}=4.66$ $\mathrm{meV}$. The thin lines in b) show the individual contributions of the A and B subsystems (i.e. even and odd layers) to the magnetizations. The presence of two Goldstone modes in the intermediate field range is an artifact of the harmonic approximation: inclusion of interaction effects would split the two modes.

cal behavior is again set by the scale $\eta_{A}^{\prime}$. (As discussed in Sec. VD, this scale vanishes at $H_{c 2}$, if the Hamiltonian has no bare $J_{z z}$.)

Once the A subsystem is ordered, the $u_{1}$ term introduces an easy-axis anisotropy for the remaining $\vec{\phi}_{B} \mathrm{U}(1)$ degrees of freedom (irrespective of the sign of $u_{1}$ ). Hence, the secondary transition at $H_{c 1}^{\prime}$ is in the Ising universality class with $z=1$. (The change from $z=2$ to $z=1$ can be understood, e.g., in terms of triplon modes: the easy-axis term couples $\tau_{+}$and $\tau_{-}$, and the resulting $2 \times 2$ matrix diagonalization results in a linearly dispersing mode at criticality. The easy-axis term is neglected in the harmonic bond-operator calculation.) The crossover with increasing energy is now from $3 \mathrm{~d}$ Ising to $2 \mathrm{~d}$ Ising to $2 \mathrm{~d}$ BEC, with crossover scales as above.

This discussion implies that, for perfect frustration, there will be a field range $H_{c 1}<H<H_{c 1}^{\prime}$ where the $B$ layers are still disordered. In this regime, the $B$ layer magnetization is zero (exponentially small) at $T=0$ (low $T$ ), respectively. This will only change at the secondary transition which occurs where the $B$ gap as function of the field reaches zero, leading to a kink e.g. in the total magnetization, see Figs. 15 and 16. Note that this kink
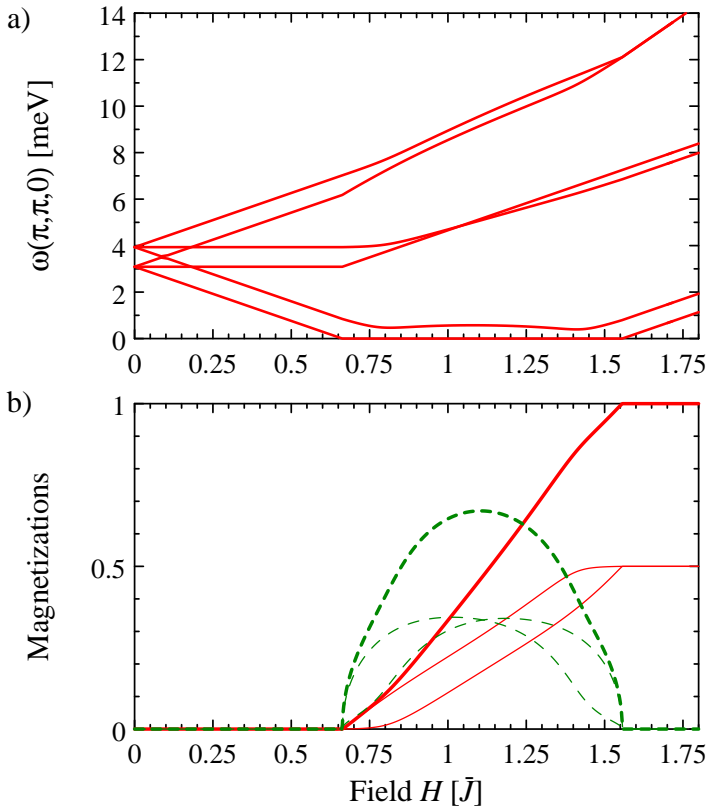

FIG. 17: a) Triplon energy gaps and b) uniform (solid) and staggered (dashed) magnetization as in Fig. 16, but now for inequivalent layers with imperfect frustration - here the secondary phase transitions are smeared out due to the proximity effect. Parameter values are: $J_{A}=4.27 \mathrm{meV}, J_{B}=5.04 \mathrm{meV}$, $J_{A}^{\prime}=J_{B}^{\prime}=0.5 \mathrm{meV}, J_{z 1}=0.05 \mathrm{meV}, J_{z 2}=0.15 \mathrm{meV}$ - the latter two values are the couplings along the two inequivalent $J_{z}$ diagonals, see Fig. $1 \mathrm{~b}$.

will also be present at finite $T$, due to the power-law onset of the secondary order parameter at the transition. The distance between the two transitions is essentially given by the difference in the zero-field gaps of the two modes $\vec{\phi}_{A}, \vec{\phi}_{B}$; small corrections arise from the density interactions between the two condensates.

As above, for imperfect frustration, the proximity effect will smear out the secondary transition, and with it the magnetization kink. The energy scale for the dimensional crossover is determined by the bare dispersion of the lowest triplon and scales as $J_{z}^{2}$.

Magnetization curves obtained using the bondoperator method are shown in Figs. 16 and 17, for perfect and imperfect frustration, respectively. For imperfect frustration, the magnetization $m_{B}$ of the $B$ subsystem is now finite above $H_{c 1}$, but depends in a non-linear fashion on the $A$ magnetization $m_{A}$ (or the field).

\section{F. Larger unit cells}

For unit cells containing more than two dimers, the number of triplon modes increases accordingly, but many qualitative features of our analysis remain valid. (i) There will be at maximum two distinct condensates, not coupled by a proximity effect in the case of perfect frustration. Hence, the number of separate sharp ordering 
transitions will not exceed two, although the number of mode gaps is larger, the reason being a proximity effect due to unfrustrated couplings. (ii) The bandwidth of the vertical dispersion for $N$ inequivalent layers will in principle be determined by $N$-th order perturbation theory; depending on the relation between the $J_{z}$ and the mode energy differences $\Delta \omega_{q}$, bandwidths between $\propto J_{z}$ and $\propto J_{z}^{N}$ are possible.

\section{APPLICATION TO BACUSI ${ }_{2} \mathrm{O}_{6}$}

After having collected information about the different scenarios, we finally come to a more detailed discussion of the behavior of $\mathrm{BaCuSi}_{2} \mathrm{O}_{6}$. First we try to summarize important experimental results: (i) The phase boundary near $H_{c 1}=23.2 \mathrm{~T}$ follows the $2 \mathrm{~d}$ power law with shift exponent $\psi=1$ down to $30 \mathrm{mK} .^{18,44}$ (ii) Zero-field neutron scattering shows multiple modes, implying inequivalent dimers. ${ }^{21}$ Together with the structural distortion below $100 \mathrm{~K}$ this suggests inequivalent layers. Further, the vertical dispersion of the modes seems tiny $(<0.1 \mathrm{meV})$ even at wavevectors away from the frustrated point. (iii) NMR experiments show the presence of two inequivalent $\mathrm{Cu}$ sites above $H_{c 1}$, with significantly different, but nonzero magnetizations. ${ }^{23}$ (iv) The magnetization curve is almost linear above $H_{c 1} \cdot{ }^{16}$ (v) Small spin anisotropies arise from magnetic dipolar interactions, but those are of order $10 \mathrm{mK}$ only. ${ }^{45}$

Points (ii) and (iii) rule out a perfect bct lattice structure with a single-dimer unit cell, Sec. V. In the following we therefore assume the inequivalence of even and odd layers, i.e., our scenario (B) of Sec. VI. Further, we will ignore the possible tendency towards incommensuration, i.e., we stick to layers with $(\pi, \pi)$ ordering. We are aware that this may not fully account for all details relevant to the material. (Note that a full characterization of the low-temperature lattice structure is still open.)

Within the scenario of inequivalent layers, perfect frustration implies two distinct condensates without proximity effect. This would invariably lead to two transitions as function of field (even at finite $T$ ), with a kink in the magnetization curve. The second transition field can be estimated from the zero-field gaps ${ }^{21}$ to be around $H_{c 1}^{\prime} \approx 27$ T. In the field range $H_{c 1}<H<H_{c 1}^{\prime}$ the magnetization in the second (non-ordered) subsystem should be exponentially small. In contrast, the NMR data ${ }^{23}$ indicate a sizeable magnetization here and hence appear inconsistent with the perfect-frustration scenario. Further, to our knowledge no secondary thermodynamic transition has been observed near $27 \mathrm{~T}$. Together, this appears to rule out perfect frustration.

We are left to consider inequivalent layers with imperfect frustration. Imperfect frustration induces a finite vertical bandwidth for all in-plane wavevectors, and it causes proximity effect, thereby smearing out the secondary ordering transition. The finite vertical bandwidth at $\vec{q}_{\|}=(\pi, \pi)$ is the relevant dimensional crossover scale

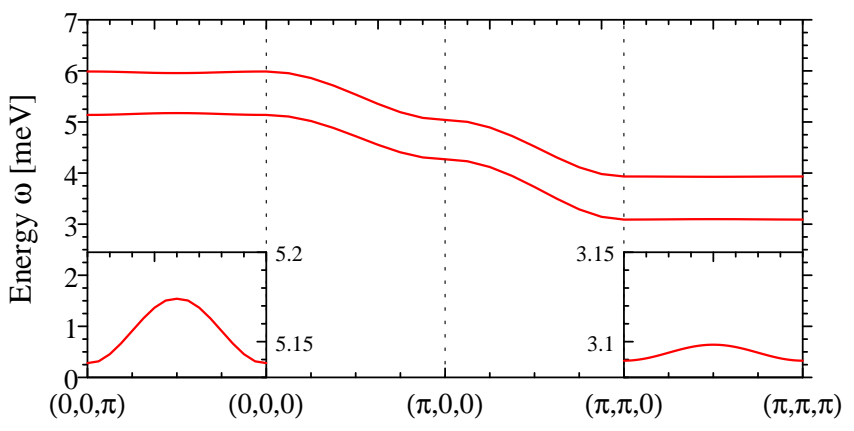

FIG. 18: Zero-field triplon dispersions for inequivalent layers with partially frustrated and modulated vertical coupling, Fig. 1c. Parameter values are: $J_{A}=4.27 \mathrm{meV}, J_{B}=5.04$ $\mathrm{meV}, J_{A}^{\prime}=J_{B}^{\prime}=0.5 \mathrm{meV}, J_{z A 1}=0.09 \mathrm{meV}, J_{z A 2}=0.11$ meV, $J_{z B 1}=0.01 \mathrm{meV}, J_{z B 2}=0.19 \mathrm{meV}$. The bandwidth along $\left(\pi, \pi, q_{z}\right)$ is reduced by a factor of 3 as compared to the situation without modulation (Fig. 11) - and can be made arbitrarily small by reducing $\left|J_{z A 1}-J_{z A 2}\right|$, but the magnetization data in the present case are essentially undistinguishable from the ones in Fig. 17 above.

below which $3 \mathrm{~d}$ behavior is observed at the phase transition. From (i), this scale is known to be less than $30 \mathrm{mK}-$ this significantly constrains the unfrustrated component, $J_{z 1}-J_{z 2}$, of the inter-layer coupling (Sec. VI A): it has to be smaller than $0.035 \mathrm{meV}$ (assuming this coupling to be vertically unmodulated; note that the square of this value is proportional to the vertical bandwidth). Taking this value, we have calculated magnetization curves which only display tiny deviations from the case of perfect frustration, Fig. 16, i.e., a pronounced magnetization kink at a second "transition" field $H_{c 1}^{\prime}$, and essentially zero magnetization in the $B$ subsystem below this field. Apparently, the proximity effect is too small to be in agreement with both the NMR and the magnetization data.

Thus, a $3 \mathrm{~d}$ scale smaller than $30 \mathrm{mK}$ is not easily compatible with the large proximity effect. To reconcile these facts, we propose the inter-layer coupling to be both frustrated and vertically modulated, see Fig. 1c. In such a situation, the unfrustrated components of the inter-layer coupling can alternate between being weak and strong, rendering a large proximity effect compatible with a tiny $3 \mathrm{~d}$ dispersion. To prove the case, we have calculated dispersions and magnetization data in a situation with $J_{z A 1}=0.11 \mathrm{meV}, J_{z A 2}=0.09 \mathrm{meV}, J_{z B 1}=0.19 \mathrm{meV}$, $J_{z B 2}=0.01 \mathrm{meV}$, i.e., the unfrustrated component of the inter-layer alternates strongly, while the frustrated component is unmodulated. With this choice, we obtain magnetization data with a sizeable proximity effect very similar to Fig. 17, but the vertical dispersion along $\left(\pi, \pi, q_{z}\right)$ [which is given by $\left(J_{z A 1}-J_{z A 2}\right)\left(J_{z B 1}-J_{z B 2}\right) / \Delta \omega_{q}$ ] is strongly reduced, Fig. 18. By reducing $\left|J_{z A 1}-J_{z A 2}\right|$ further, this $3 \mathrm{~d}$ scale gets arbitrarily small, without significant changes in the magnetizations. (Note that the precise shape of the magnetization curve will be influenced by logarithmic corrections not captured by our harmonic 
approximation, but the presence or absence of a rather sharp kink will be unaffected. As stated above, we never find a field-independent ratio of the two magnetizations, $m_{A} / m_{B}$, and attribute the experimental observation ${ }^{23}$ of $m_{A} / m_{B} \approx 5$ to a coincidence related to proximity effect and logarithmic corrections.)

In summary, within the scenario of modulated vertical couplings we can easily get consistency between two key observations: a tiny $3 \mathrm{~d}$ scale and a large proximity effect. We note that a modulation of the unfrustrated part of the coupling is required for both phenomena; the largest unfrustrated coupling is of order $0.2 \mathrm{meV}$ (Fig. 18) and hence relevant for the magnetism not only at lowest $T$. There are more free parameters in the proposed model: an additional modulation of the frustrated part will mainly reduce the bandwidth along $\left(0,0, q_{z}\right)$ - here future neutron scattering experiments will help to put bounds on these couplings. In the absence of further experimental information on the $3 \mathrm{~d}$ dispersion scales we refrain from a more detailed fitting of the experimental data.

Let us finally comment on the transition temperature $T_{\mathrm{N}}$. In a general quasi-2d XY magnet with some (unfrustrated) inter-layer coupling $J_{z}, T_{\mathrm{N}}$ will sensitively depend on $J_{z}$ : For $J_{z} \rightarrow 0, T_{\mathrm{N}}$ is given by the Kosterlitz-Thouless temperature $T_{\mathrm{KT}}$, but a finite $J_{z}$ will induce a strong (logarithmic) enhancement to that, due to the exponentially large correlation length above the $2 \mathrm{~d}$ Kosterlitz-Thouless transition. For a partially frustrated inter-layer coupling, both the unfrustrated and frustrated parts will contribute to $T_{\mathrm{N}}$, but the detailed behavior may be complicated (see the discussion in Sec. VE), and a quantitative calculation of $T_{\mathrm{N}}$ is beyond the scope of the paper. Applied to $\mathrm{BaCuSi}_{2} \mathrm{O}_{6}$, we believe that the rather small value of the unfrustrated $3 \mathrm{~d}$ dispersion scale $(\lesssim 30 \mathrm{mK})$ may well be compatible with the observed $T_{\mathrm{N}}$ (which is roughly twice as large as the expected $T_{\mathrm{KT}}$ ), as the frustrated part of the inter-layer coupling is likely significantly larger $(\lesssim 0.5$ $\mathrm{K})$ and enhances $T_{\mathrm{N}}$ as well.

\section{CONCLUSIONS}

We have studied quantum dimer magnets on layered lattices of the bct type. Guided by experimental results on $\mathrm{BaCuSi}_{2} \mathrm{O}_{6}$, we have discussed two distinct routes towards magnetism with reduced dimensionality: (A) an ideal bct lattice with perfect frustration, and (B) a distorted lattice with inequivalent layers and possibly broken frustration. While the situations with perfect frustration are theoretically fascinating, the material $\mathrm{BaCuSi}_{2} \mathrm{O}_{6}$ likely falls into class (B) without perfect frustration. There, dimensional reduction primarily arises from a layer "mismatch": vertical magnon hopping proceeds via a second-order process of strength $J_{z}^{2} / \Delta \omega_{q}$ (where $\Delta \omega_{q}$ is the magnon energy difference between even and odd layers). In addition, we propose that a further reduction of the $3 \mathrm{~d}$ energy scale originates from a modulation of $J_{z}$ along the $c$ axis, with the unfrustrated part being small on every second link. This scenario allows to reconcile the presence of inequivalent layers ${ }^{21,23}$ and the tiny $3 \mathrm{~d}$ energy scale ${ }^{18}$ with the rather large magnetic proximity effect. ${ }^{23}$

For the case of perfect frustration, a number of theoretical challenges are left for future work: Those include (i) a more detailed study of the dimensional crossover behavior in the quantum critical regime, and (ii) the fate of the order-from-disorder mechanism at elevated temperatures and the nature of the finite-temperature transition, including a reliable estimate of the Néel temperature.

\section{Acknowledgments}

We thank C. Batista, I. Fischer, M. Garst, V. N. Kotov, F. Mila, J. Mydosh, R. Stern and in particular B. Normand, A. Rosch, Ch. Rüegg, and J. Schmalian for discussions, furthermore J. Sirker and O. Sushkov for pointing out the role of vertex corrections in the bondoperator approach in finite fields, Ch. Rüegg for sharing unpublished experimental data, and C. Vojta for assistance and support. This research was supported by the DFG through Research Unit 960 and SFB 608.

\section{APPENDIX A: BOND OPERATORS BEYOND THE HARMONIC APPROXIMATION}

In this appendix we present details of the paramagnetic bond-operator approach introduced in Sec. IV B - this is a generalization of the formalism of Ref. 28 to finite fields. An additional generalization to finite temperature is possible as well, but we shall not follow that here.

The Hamiltonian is given by $\mathcal{H}=\mathcal{H}_{2}+\mathcal{H}_{4}+\mathcal{H}_{U}$, with $\mathcal{H}_{2}$ and $\mathcal{H}_{U}$ given in (15) and (20), respectively. The quartic term $\mathcal{H}_{4}=\mathcal{H}_{4 \|}+\mathcal{H}_{4 z}$ is

$$
\begin{aligned}
\mathcal{H}_{4 \|} & =\frac{J^{\prime}}{2} \sum_{\langle i j\rangle n} h_{4}(i n ; j n), \\
\mathcal{H}_{4 z} & =\frac{J_{4 z}}{4} \sum_{i \Delta n} h_{4}(i n ; i+\Delta, n+1),
\end{aligned}
$$

with dimer site indices (in) as in Eq. (1), $J_{4 z}=J_{z}^{11}+$ $J_{z}^{22}+J_{z}^{12}+J_{z}^{21}$, and $h_{4}$ given by

$$
\begin{aligned}
h_{4}(i ; j)= & {\left[t_{j 0}^{\dagger} t_{i 0}\left(t_{i+}^{\dagger} t_{j+}+t_{i-}^{\dagger} t_{j-}\right)\right.} \\
& \left.-t_{i 0} t_{j 0}\left(t_{i+}^{\dagger} t_{j-}^{\dagger}+t_{i-}^{\dagger} t_{j+}^{\dagger}\right)+\text { h.c. }\right] \\
& +\left(t_{i+}^{\dagger} t_{i+}-t_{i-}^{\dagger} t_{i-}\right)\left(t_{j+}^{\dagger} t_{j+}-t_{j-}^{\dagger} t_{j-}\right) .
\end{aligned}
$$

In principle, there is also a cubic term,

$$
\mathcal{H}_{3 z}=\sum_{i \Delta n}\left[\frac{J_{3 z}^{+}}{4} h_{3}(i n ; i+\Delta, n+1)+\frac{J_{3 z}^{-}}{4} h_{3}(i+\Delta, n+1 ; i n)\right],
$$


with $J_{3 z}^{ \pm}=J_{z}^{11}-J_{z}^{22} \pm\left(J_{z}^{12}-J_{z}^{21}\right)$ and

$$
\begin{aligned}
h_{3}(i ; j)= & \sum_{i \Delta n}\left(\left(t_{i 0}+t_{i 0}^{\dagger}\right)\left(t_{j-}^{\dagger} t_{j+}-t_{j+}^{\dagger} t_{j-}\right)\right. \\
& \left.+\left[\left(t_{i+}+t_{i-}^{\dagger}\right)\left(t_{j 0}^{\dagger} t_{j-}-t_{j+}^{\dagger} t_{j 0}\right)+\text { h.c. }\right]\right) .
\end{aligned}
$$

We neglect it as its inclusion would not qualitatively affect our conclusions: Because of the geometric frustration, self-energy contributions deriving from $\mathcal{H}_{3 z}$ which are $\propto J_{3 z}^{2}$ (Fig. $4 \mathrm{~d}$ ) give no $q_{z}$-dependent correction to the triplon dispersion for $\vec{q}_{\|}=(\pi, \pi)$.

The effect of the local and non-retarded interaction $\mathcal{H}_{U}$ can be captured by a ladder summation, leading to a four-point vertex

$$
\Gamma_{\alpha \beta, \alpha \beta}(\vec{k}, \omega)=-\left(\frac{1}{N} \sum_{\vec{p}} \frac{u_{\vec{p}}^{2} u_{\vec{k}-\vec{p}}^{2}}{\omega-\omega_{\vec{p} \alpha}-\omega_{\vec{k}-\vec{p}, \beta}}\right)^{-1}
$$

where $N$ is the number of lattice sites. Here, all anomalous scattering vertices have been neglected, which is justified if the number of triplet bosons is small (Brueckner approximation). (Note that the Bogoliubov coefficients $u, v$ do not depend on the Zeeman index, see below.)

To leading order, the normal self energy from $\mathcal{H}_{U}$ is given by the sum of Hartree and Fock diagrams (Fig. 4a):

$$
\begin{aligned}
\Sigma_{\alpha}^{U}(\vec{k}, \omega) & =\Sigma_{\alpha \alpha}(\vec{k}, \omega)+\sum_{\beta} \Sigma_{\alpha \beta}(\vec{k}, \omega) \\
\Sigma_{\alpha \beta}(\vec{k}, \omega) & =\frac{1}{N} \sum_{\vec{q}} v_{\vec{q} \beta}^{2} \Gamma_{\alpha \beta, \alpha \beta}\left(\vec{k}+\vec{q}, \omega-\omega_{\vec{q} \beta}\right) .(
\end{aligned}
$$

For the bct lattice, this self-energy will induce a triplon dispersion along $\left(\pi, \pi, q_{z}\right)$ of order $J_{z}^{6}$, with the leading process being the one in Fig. 3b2 (the scattering amplitude from $\mathcal{H}_{U}$ is of order unity).

In addition to the hard-core interaction $\mathcal{H}_{U}$, we take into account the quartic $t$ terms in $\mathcal{H}_{4}$ in a mean-field approximation, Fig. 4b. This gives additional self energies:

$$
\begin{aligned}
& \Sigma_{\alpha}^{n}(\vec{k})=\frac{2}{N} \sum_{\vec{q}}\left(J^{\prime} \gamma_{\vec{k} \|} \gamma_{\vec{q} \|}+J_{4 z} \gamma_{\vec{k} z} \gamma_{\vec{q} z}\right) \sum_{\beta \neq \alpha} v_{\vec{q}}^{2} \\
& \Sigma_{\alpha}^{a}(\vec{k})=-\frac{2}{N} \sum_{\vec{q}}\left(J^{\prime} \gamma_{\vec{k} \|} \gamma_{\vec{q} \|}+J_{4 z} \gamma_{\vec{k} z} \gamma_{\vec{q} z}\right) \sum_{\beta \neq \alpha} u_{\vec{q}} v_{\vec{q}}
\end{aligned}
$$

with $\gamma_{\vec{q} \|}$ and $\gamma_{\vec{k} z}$ defined in Eq. (16). While these meanfield terms do not contribute to the dispersion along $\left(\pi, \pi, q_{z}\right)$, higher-order processes in $\mathcal{H}_{4 z}$ do so. Remarkably, the second-order self energy, Fig. 4c, turns out to give the leading contribution to the vertical dispersion, scaling with $J_{z}^{4}\left(J_{z}^{2}\right.$ from the vertices and $J_{z}^{2}$ for vertical hopping on one internal line). Therefore we include a contribution to $\Sigma_{\alpha}^{n}(\vec{k})$, which is of second order in $\mathcal{H}_{4 z}$, in addition to the Hartree-like terms above. The evaluation of the diagram in Fig. 4c gives (here displayed for

$$
\begin{aligned}
& \alpha=+): \\
& \sum_{+}^{n, \operatorname{extra}}(\vec{k}, \omega)=J_{4 z}^{2} \frac{4}{N^{2}} \sum_{\vec{p} \vec{q}} \gamma_{\vec{k}-\vec{q}, z}^{2}\left[f_{\vec{k} \vec{p} \vec{q} \omega}(+, 0,0)\right. \\
& \left.\quad+2 f_{\vec{k} \vec{p} \vec{q} \omega}(0,0,-)+2 f_{\vec{k} \vec{p} \vec{q} \omega}(+,+,+)+f_{\vec{k} \vec{p} \vec{q} \omega}(+,-,-)\right]
\end{aligned}
$$

with

$$
f_{\vec{k} \vec{p} \vec{q} \omega}(\alpha, \beta, \gamma)=\frac{u_{\vec{k}+\vec{p}-\vec{q}}^{2} u_{\vec{q}}^{2} v_{\vec{p}}^{2}}{\omega-\omega_{\vec{k}+\vec{p}-\vec{q}, \alpha}-\omega_{\vec{q}, \beta}-\omega_{\vec{p}, \gamma}} .
$$

The normal self energies $\Sigma^{U}$ and $\Sigma^{n}$ add up, $\bar{\Sigma}^{n}(\vec{k})=$ $\Sigma^{n}(\vec{k})+\Sigma^{U}(\vec{k}, \omega=0)$. With the self energies we can define renormalized coefficients $\tilde{A}_{\vec{k}}, \tilde{B}_{\vec{k}}$ with

$$
\tilde{A}_{\vec{k}}=A_{\vec{k}}+\bar{\Sigma}^{n}(\vec{k}), \tilde{B}_{\vec{k}}=B_{\vec{k}}+\Sigma^{a}(\vec{k}) .
$$

Solving the coupled Dyson equations for the normal and anomalous Green's functions yields the renormalized spectrum for the quasiparticles,

$$
\Omega_{\vec{k} \alpha}=Z_{\vec{k}} \sqrt{\tilde{A}_{\vec{k}}^{2}-\tilde{B}_{\vec{k}}^{2}}-\alpha H,
$$

with a quasiparticle weight

$$
Z_{\vec{k}}^{-1}=1-\left.\frac{\partial \Sigma^{U}(\vec{k}, \omega)}{\partial \omega}\right|_{\omega=0},
$$

and the renormalized Bogoliubov coefficients:

$$
\begin{aligned}
U_{\vec{k}}^{2}, V_{\vec{k}}^{2} & = \pm \frac{1}{2}+\frac{\tilde{A}_{\vec{k}}}{2 \sqrt{\tilde{A}_{\vec{k}}^{2}-\tilde{B}_{\vec{k}}^{2}}}, \\
U_{\vec{k}} V_{\vec{k}} & =-\frac{\tilde{B}_{\vec{k}}}{2 \sqrt{\tilde{A}_{\vec{k}}^{2}-\tilde{B}_{\vec{k}}^{2}}} .
\end{aligned}
$$

A fully self-consistent solution of the problem requires the substitutions

$$
\omega_{\vec{k} \alpha} \rightarrow \Omega_{\vec{k} \alpha}, \quad u_{\vec{k}} \rightarrow \sqrt{Z_{\vec{k}}} U_{\vec{k}}, \quad v_{\vec{k}} \rightarrow \sqrt{Z_{\vec{k}}} V_{\vec{k}}
$$

in the equations (A5,A6, A7, A9).

The reader may wonder about a possible $\alpha$ (spin) dependence of the self energies and the Bogoliubov coefficients. Indeed, the naive perturbative approach sketched here results in such a spin dependence, which consequently also yields a renormalization of the magnetic field (or the $g$ factor). Physically, such a result is incorrect, as the $z$ component of the total spin is conserved. This conservation law can be formally implemented by a corresponding Ward identity, resulting in appropriate vertex corrections. Here, a consistent result can be obtained by implementing a finite field through the replacement $\omega \rightarrow \omega+\alpha H$ everywhere. This implies that the selfenergies need to be evaluated at energy $\alpha H$ instead of zero. Doing this, the self energies and Bogoliubov coefficients remain $\alpha$ independent. ${ }^{46}$ (Practically, only the $\alpha=0$ self energy needs to be evaluated.) 


\section{APPENDIX B: SCATTERING AMPLITUDE FOR BETHE-SALPETER EQUATION}

We consider a biquadratic coupling between adjacent layers, i.e. a simplified version of Eq. (4):

$$
\mathcal{H}_{\text {coll }}=\frac{J_{\text {coll }}}{4} \sum_{i \Delta n m m^{\prime}}\left(\vec{S}_{i n m} \cdot \vec{S}_{i+\Delta, n+1, m^{\prime}}\right)^{2},
$$

rewrite it in terms of bond operators and extract from the quartic terms the following contribution to the bare scattering amplitude

$$
\begin{aligned}
M_{\alpha \beta, \gamma \delta}^{\mathrm{coll}}= & \frac{J_{\mathrm{coll}}}{2}\left[\left(\delta_{\alpha \gamma} \delta_{\beta \delta}+\delta_{\alpha \beta} \delta_{\gamma \delta}\right) \gamma_{\vec{p}+\vec{q}, z}\right. \\
& +\left(\delta_{\alpha \delta} \delta_{\beta \gamma}+\delta_{\alpha \beta} \delta_{\gamma \delta}\right) \gamma_{\vec{p}-\vec{q}, z} \\
& \left.+\left(\delta_{\alpha \gamma} \delta_{\beta \delta}+\delta_{\alpha \delta} \delta_{\beta \gamma}\right) \gamma_{\vec{Q} z}\right]
\end{aligned}
$$

where we employ the notation of Ref. 37. This adds to the result deriving from $\mathcal{H}_{4}$ and $\mathcal{H}_{U}$,

$$
\begin{aligned}
M_{\alpha \beta, \gamma \delta}^{\text {other }}= & 2\left(\delta_{\alpha \gamma} \delta_{\beta \delta}-\delta_{\alpha \beta} \delta_{\gamma \delta}\right)\left(J^{\prime} \gamma_{\vec{p}+\vec{q}, \|}+J_{4 z} \gamma_{\vec{p}+\vec{q}, z}\right) \\
& +2\left(\delta_{\alpha \delta} \delta_{\beta \gamma}-\delta_{\alpha \beta} \delta_{\gamma \delta}\right)\left(J^{\prime} \gamma_{\vec{p}-\vec{q}, \|}+J_{4 z} \gamma_{\vec{p}-\vec{q}, z}\right) \\
& +U\left(\delta_{\alpha \delta} \delta_{\beta \gamma}+\delta_{\alpha \delta} \delta_{\beta \gamma}\right) .
\end{aligned}
$$

Altogether, the scattering amplitude in the singlet $(S=$ 0 ) channel is

$$
\begin{aligned}
M^{(0)}= & \frac{1}{3} \delta_{\alpha \beta} \delta_{\gamma \delta}\left(M_{\alpha \beta, \gamma \delta}^{\text {other }}+M_{\alpha \beta, \gamma \delta}^{\text {coll }}\right) \\
= & -4 J^{\prime}\left(\gamma_{\vec{p}+\vec{q}, \|}+\gamma_{\vec{p}-\vec{q}, \|}\right)-4 J_{4 z}\left(\gamma_{\vec{p}+\vec{q}, z}+\gamma_{\vec{p}-\vec{q}, z}\right) \\
& +2 U+J_{\text {coll }}\left(2 \gamma_{\vec{p}+\vec{q}, z}+2 \gamma_{\vec{p}-\vec{q}, z}+\gamma_{\vec{Q} z}\right) .
\end{aligned}
$$

Interestingly, the quartic scattering terms arising from $J_{4 z}$ and $J_{\text {coll }}$ are of essentially identical form (although the Hamiltonians are different). Consequently, both $\mathcal{H}_{4 z}$ and $\mathcal{H}_{\text {coll }}$ cause similar triplon bound states.
1 J. A. Hertz, Phys. Rev. B 14, 1165 (1976).

2 T. Moriya, Spin Fluctuations in Itinerant Electron Magnetism, Springer-Verlag, Berlin (1985); T. Moriya and T. Takimoto, J. Phys. Soc. Jpn. 64, 960 (1995).

3 A. J. Millis, Phys. Rev. B, 48, 7183 (1993).

4 S. Sachdev, Quantum Phase Transitions, Cambridge University Press, Cambridge (1999).

5 M. Vojta, Rep. Prog. Phys. 66, 2069 (2003).

${ }^{6}$ H. v. Löhneysen, A. Rosch, M. Vojta, and P. Wölfle, Rev. Mod. Phys. 79, 1015 (2007).

7 H. v. Löhneysen, J. Phys. Cond. Matter 8, 9689 (1996).

8 O. Stockert, H. v. Löhneysen, A. Rosch, N. Pyka, and M. Loewenhaupt, Phys. Rev. Lett. 80, 5627 (1998).

9 O. Trovarelli, C. Geibel, S. Mederle, C. Langhammer, F. M. Grosche, P. Gegenwart, M. Lang, G. Sparn, and F. Steglich, Phys. Rev. Lett. 85, 626 (2000).

10 Q. Si, S. Rabello, K. Ingersent, and J. L. Smith, Nature 413, 804 (2001) and Phys. Rev. B 68, 115103 (2003).

11 K. Takatsu, W. Shiramura, and H. Tanaka, J. Phys. Soc. Jpn. 66, 1611 (1997).

12 N. Cavadini, G. Heigold, W. Henggeler, A. Furrer, H.-U. Güdel, K. Krämer, and H. Mutka, Phys. Rev. B 63, 172414 (2001).

13 T. Nikuni, M. Oshikawa, A. Oosawa, and H. Tanaka, Phys. Rev. Lett. 84, 5868 (2000).

14 Ch. Rüegg, N. Cavadini, A. Furrer, H.-U. Güdel, K. Krämer, H. Mutka, A. Wildes, K. Habicht, and P. Vorderwisch, Nature 423, 62 (2003).

15 Y. Sasago, K. Uchinokura, A. Zheludev, and G. Shirane, Phys. Rev. B 55, 8357 (1997).

16 M. Jaime, V. F. Correa, N. Harrison, C. D. Batista, N. Kawashima, Y. Kazuma, G. A. Jorge, R. Stern, I. Hein- maa, S. A. Zvyagin, Y. Sasago, and K. Uchinokura, Phys. Rev. Lett. 93, 087203 (2004).

17 S. E. Sebastian, P. A. Sharma, M. Jaime, N. Harrison, V. Correa, L. Balicas, N. Kawashima, C. D. Batista, and I. R. Fisher, Phys. Rev. B 72, 100404(R) (2005).

18 S. E. Sebastian, N. Harrison, C. D. Batista, L. Balicas, M. Jaime, P. A. Sharma, N. Kawashima, and I. R. Fisher, Nature 441, 617 (2006).

19 C. D. Batista, J. Schmalian, N. Kawashima, S. E. Sebastian, N. Harrison, M. Jaime, and I. R. Fisher, Phys. Rev. Lett. 98, 257201 (2007).

20 O. Rösch and M. Vojta, Phys. Rev. B 76, 180401(R) (2007).

${ }^{21}$ Ch. Rüegg, D. F. McMorrow, B. Normand, H. M. Ronnow, S. E. Sebastian, I. R. Fisher, C. D. Batista, S. Gvasaliya, Ch. Niedermayer, and J. Stahn, Phys. Rev. Lett. 98, 017202 (2007).

22 E. C. Samulon, Z. Islam, S. E. Sebastian, P. B. Brooks, M. K. McCourt, Jr., J. Ilavsky, and I. R. Fisher, Phys. Rev. B 73, 100407(R) (2006).

23 S. Krämer, R. Stern, M. Horvatic, C. Berthier, T. Kimura, and I. R. Fisher, arXiv:0704.0888 (2007).

24 M. Maltseva and P. Coleman, Phys. Rev. B 72, 174415 (2005).

25 A. W. Sandvik and D. J. Scalapino, Phys. Rev. Lett. 72, 2777 (1994).

26 A. W. Sandvik, A. V. Chubukov, and S. Sachdev, Phys. Rev. B 51, 16483 (1995).

27 M. Troyer and S. Sachdev, Phys. Rev. Lett. 81, 5418 (1998).

28 V. N. Kotov, O. P. Sushkov, Zheng Weihong, and J. Oitmaa, Phys. Rev. Lett. 80, 5790 (1998). 
29 T. Sommer, M. Vojta, and K. W. Becker, Eur. Phys. J. B 23, 329 (2001).

30 M. B. Stone, I. A. Zaliznyak, T. Hong, C. L. Broholm, and D. H. Reich, Nature 440, 187 (2006).

31 A. Kolezhuk and S. Sachdev, Phys. Rev. Lett. 96, 087203 (2006); A. L. Chernyshev and M. E. Zhitomirsky, Phys. Rev. Lett. 97, 207202 (2006).

32 S. Sachdev and R. N. Bhatt, Phys. Rev. B 41, 9323 (1990).

33 P. V. Shevchenko, A. W. Sandvik, and O. P. Sushkov, Phys. Rev. B 61, 3475 (2000).

34 M. Matsumoto, B. Normand, T. M. Rice, and M. Sigrist, Phys. Rev. Lett. 89, 077203 (2002).

35 T. Giamarchi and A. Tsvelik, Phys. Rev. B 59, 11398 (1999).

36 R. Eder, Phys. Rev. B 57, 12832 (1998).

37 O. P. Sushkov and V. N. Kotov, Phys. Rev. Lett. 81, 1941 (1998); V. N. Kotov, O. P. Sushkov, and R. Eder, Phys. Rev. B 59, 6266 (1999).

38 N. Read and S. Sachdev, Phys. Rev. Lett. 62, 1694 (1989).

39 E. Rastelli, S. Sedazzari, and A. Tassi, J. Phys. C: Solid State Phys. 18, 353 (1985), J. Phys. Cond. Matter 1, 4735 (1989).
40 E. Rastelli, S. Sedazzari, and A. Tassi, J. Phys. Cond. Matter 2, 8935 (1990).

41 T. Yildirim, A. B. Harris, and E. F. Shender, Phys. Rev. B 53, 6455 (1996).

42 O. A. Starykh and L. Balents, Phys. Rev. Lett. 98, 077205 (2007).

43 C. Yasuda, S. Todo, K. Hukushima, F. Alet, M. Keller, M. Troyer, and H. Takayama, Phys. Rev. Lett. 94, 217201 (2005); A. Praz, C. Mudry, and M. B. Hastings, Phys. Rev. B 74, 184407 (2006); D. X. Yao, and A. W. Sandvik, arXiv:cond-mat/0606341 (2006).

44 M. Horvatic, C. Berthier, F. Tedoldi, A. Comment, M. Sofin, M. Jansen, and R. Stern, Prog. Theor. Phys. Suppl. 159, 106 (2005).

45 S. E. Sebastian, P. Tanedo, P. A. Goddard, S.-C. Lee, A. Wilson, S. Kim, S. Cox, R. D. McDonald, S. Hill, N. Harrison, C. D. Batista, and I. R. Fisher, Phys. Rev. B 74, 180401(R) (2006).

46 J. Sirker, A. Weisse, and O. P. Sushkov, J. Phys. Soc. Jpn. 74 Suppl.,129 (2005). 